\title{
Low geo-effectiveness of fast halo CMEs related to the 12 X-class flares in 2002
}

\author{
B. Schmieder ${ }^{1,2,3 *}$, R.-S. Kim ${ }^{2,4}$, B. Grison ${ }^{5}$, K. Bocchialini ${ }^{6}$, R.-Y. Kwon ${ }^{2}$, S. \\ Poedts $^{3,7}$, P. Démoulin ${ }^{1}$ \\ ${ }^{1}$ LESIA, Observatoire de Paris, PSL Research University, CNRS Sorbonne Université, Univ. Paris 06, \\ Univ. Paris Diderot, Sorbonne Paris Cité, 5 place Jules Janssen, F-92195 Meudon, France \\ ${ }^{2}$ Korea Astronomy and Space Science Institute, 776 Daedeok-daero, Yuseong-gu, Daejeon 34055, Korea \\ ${ }^{3}$ Dept. of Mathematics, KU Leuven, Celestijnenlaan 200B, 3001 Leuven, Belgium \\ ${ }^{4}$ University of Science and Technology, 217 Gajeong-ro, Yuseong-gu, Daejeon, 34113. Korea \\ ${ }^{5}$ Department of Space Physics, Institute of Atmospheric Physics of the Czech Academy of Sciences, Bocni \\ II, 1401, 14100 Prague 4, Czech Republic \\ ${ }^{6}$ Institut d'Astrophysique Spatiale, Univ. Paris-Sud, CNRS, Université Paris-Saclay, Bâtiment 121, 91405 \\ Orsay CEDEX, France \\ ${ }^{7}$ Group of Astrophysics, Institute of Physics, University of Maria Curie-Skłodowska, ul. Radziszewskiego \\ 10, PL-20-031 Lublin, Poland
}

\section{Key Points:}

- We study all the X-class flares and associated CMEs in 2002 (12 cases)

- None of the six halo CMEs and shocks evoked strong geomagnetic disturbances possibly due to the weak southward magnetic field components of the ICMEs.

- The favorable solar proxies (large sunspot, fast halo CME, ...) were not sufficient to accurately forecast the geo-effectiveness of these CMEs.

*visitor in KASI 


\begin{abstract}
It is generally accepted that extreme space weather events tend to be related to strong flares and fast halo coronal mass ejections CMEs. In the present paper, we carefully identify the chain of events from the Sun to the Earth induced by all $12 \mathrm{X}$-class flares that occurred in 2002. In this small sample, we find an unusual high rate $(58 \%)$ of solar sources with a longitude larger than 74 degrees. Yet, all $12 \mathrm{X}$-class flares are associated with at least one CME. The fast halo CMEs (50\% ) are related to interplanetary CMEs (ICMEs) at $\mathrm{L} 1$ and weak Dst minimum values $(>-51 \mathrm{nT})$; while $5(41 \%)$ of the $12 \mathrm{X}$-class flares are related to solar proton events (SPE).

We conclude that: (i) All twelve analyzed solar events, even those associated with fast halo CMEs originating from the central disk region, and those ICMEs and SPEs were not very geo-effective. This unexpected result demonstrates that the suggested events in the chain (fast halo CME, X-class flares, central disk region, ICME, SPE) are not infallible proxies for geo-effectiveness. (ii) The low value of integrated and normalized southward component of the IMF $\left(B_{z}^{*}\right)$ may explain the low geo-effectiveness for this small sample. In fact, $B_{z}^{*}$ is well correlated to the weak Dst and low auroral electrojet (AE) activity. Hence, the only space weather impact at Earth in 2002 we can explain is based on $B_{z}^{*}$ at L1.
\end{abstract}

\title{
1 Introduction
}

It is generally recognized that extreme space weather events are often related to large, mature sunspot groups. Such turbulent and highly complex solar active regions (ARs) have a large amount of "free" (non-potential) magnetic energy, which can be released during flares. Flares irradiate large amounts of thermal energy, up to $10^{33} \mathrm{ergs}$ (Aulanier et al., 2013). Intense X-class flares have irradiance values (E) exceeding $10^{-4} \mathrm{~W} / \mathrm{m}^{2}$. They even affect the ionosphere due to their X-ray emission flux which can perturb the geo-localization systems (Tsurutani et al., 2009). Fast CMEs are usually associated with flares and are enormous eruptions of plasma (up to $10^{13}-10^{16} \mathrm{~g}$ ) and magnetic field into the interplanetary space at velocities up to several thousand $\mathrm{km} / \mathrm{s}$ which interact with the solar wind and often also with the terrestrial magnetosphere (Denig et al., 2015). The kinetic energy of CMEs reaches up to $10^{32}$ ergs. Clearly, full front-side halo CMEs have more potential to arrive at the Earth a few days after their launch (Kim et al., 2005). Magnetic reconnection during flare onset and shock waves driven by CMEs can both generate solar energetic particles (SEPs), creating sporadic concentrations at Earth known as Solar Particle Events (SPEs) which are characterized by enhancements of several orders of magnitude above background levels, e.g. for protons energies vary from less than $1 \mathrm{MeV}$ to over $1 \mathrm{GeV}$ in extreme cases (Lario \& Simnett, 2004; Nitta et al., 2006; Cliver \& Ling, 2007; Cane et al., 2010; Kim et al., 2015). CMEs surfing on the solar wind are called interplanetary CMEs (ICMEs). When they are embedded in a strong magnetic field with a smooth rotation, and have a low plasma beta and proton temperature, they are called magnetic clouds (MC) (Burlaga et al., 1981). Consequently, flare-related CMEs are key in current space weather operational forecasting systems considering their potentially severe impact on Earth, electric power blackouts, and damage to spacecraft instrumentation. There are considerable research efforts underway to improve our understanding of CMEs to provide feedback to operational space weather forecasting infrastructure (Vourlidas et al., 2019). The predicted time of occurrence of geo-effective events in the Earth environment depends on the effects studied:

- The common transit time from Sun to Earth for CME/ICMEs and coronal hole high speed wind streams can be estimated up to 2 to 4 days (Gopalswamy et al., 2007; Scolini et al., 2018), i.e. only after this time the related effects such as geomagnetic storms, energetic particle enhancements, and geomagnetic induced currents (GIC) are measured at Earth (Vanhamäki et al., 2013). 
- Impulsive SPEs (from a few $\mathrm{keV}$ ) up to relativistic energies (few $\mathrm{GeV}$ ), accelerated by magnetic reconnection driven processes during the impulsive phases of solar flares, have a transit time as low as ten minutes while large gradual SPE events are produced by fast CME driven shock waves and can last a few hours or even days depending on their trajectories and their orientation in the interplanetary magnetic field (IMF) (Zurbuchen \& Richardson, 2006). SPEs can penetrate down to the upper atmosphere leading to solar array degradation, single event effects in electronics and potential health risks for manned spaceflight and air travelers (Lario \& Simnett, 2004).

Many different methods exist to predict such events based on the determination of the initial speed, direction, shape and evolution of CMEs through the inner heliosphere and in the IMF (Cliver et al., 1990; Tsurutani et al., 1988, 1992; Tsurutani, Wu, et al., 2003). The extreme space weather events which arrive at Earth eight minutes after flares are not predictable without a good prediction of the occurrence of large flares. The largest 'extreme' events are exceptional events occurring once or even less than once during a solar cycle (Wimmer-Schweingruber, 2014; Denig et al., 2015). Solar energetic particles of moderate strength occur more frequent, as well as geomagnetic storms. Geomagnetic storms driven by ICMEs may be predicted, based on the CME characteristics, between two to four days before arriving in the Earth environment. It is difficult nevertheless to get a better forecasting accuracy than $50 \%$ taking into account the different CME parameters (Vršnak et al., 2005; Gopalswamy et al., 2007; Richardson \& Cane, 2010; Bein et al., 2012; Jang et al., 2017). Moreover, interaction of ICMEs and interplanetary structures, e.g., high speed streams, are also reported to be drivers of severe geomagnetic activity (Gonzalez et al., 1996; Y. M. Wang et al., 2003; Xie et al., 2006; Lugaz et al., 2017). Geomagnetic storms have been intensively studied by Cliver et al. (1990); Tsurutani et al. (1992). The $B_{z}$ component of the IMF is well correlated to the storm Dst value (Wu \& Lepping, 2002). Moreover, ICMEs generating long periods of strong southward (negative) $B_{z}$ can interact with the magnetosphere magnetic field and initiate geomagnetic storms (Tsurutani et al., 1988; Gosling, 1990). This effect is even increasing with high speed solar wind or fast ICMEs.

Statistical approaches are important to detect associations and correlations in the chain of events from Sun to Earth. Richardson and Cane (2010) listed 300 near Earth ICMEs and summarized their basic properties and their related geomagnetic effects. Gopalswamy et al. (2007) listed 378 halo CMEs during solar cycle 23 and their geo-effectiveness (characterized by $\mathrm{Dst}_{\min }$ ). These authors confirmed that halo CMEs originating near the central meridian have a better chance to be highly geo-effective. They also noted that the geoeffectiveness rate is particularly low in 1999 and 2002 (the beginning and the end of the maximum solar phase of solar cycle 23). Cane et al. (2010) examined all the energetic particle events occurring during solar cycle 23 and suggested that their main origins are flares with short or long duration depending on whether they were associated with CMEs or not. Cane et al. (2010); Dierckxsens et al. (2015); Miteva, Samwel, and Costa-Duarte (2018); Salas-Matamoros and Klein (2015) concentrated their studies on the SEP events during solar cycle 23. It was found that the probability of an SEP event occurring is higher for intense flares, high speed CMEs and sources with western longitude. It was also confirmed that there is a strong relationship between halo CMEs and SPEs (Chandra et al., 2013). Kim et al. (2015) classified the main SPEs occurring during solar cycle 23 in four categories according to their onset times compared with the flare peak and the delays existing between the different channels of energy. Nieves-Chinchilla et al. (2019) provided a full catalog of the ICMEs observed between 1995 and 2015. These authors investigated the magnetic field configuration associated with 353 ICMEs in order to validate their flux-rope structure. The Jian, Russell, Luhmann, and Skoug (2006) catalog gathers most of the ICMEs identified during this time period. 
However, most of the studies do not consider the whole chain of events from the solar surface to the Earth, as this is rather difficult when starting from the solar source as opposed to starting from the ICME or its geo-effectiveness. Instead, they focus merely on some parts of the chain or study only individual extreme events (Tsurutani, Gonzalez, et al., 2003; Denig et al., 2015; Kim et al., 2005, 2015; Schmieder, 2018). Therefore, the physical chain of processes is usually not really studied as a whole. A scatter plot diagram between two events in the chain might give a higher probability of their correlation but this does not explain why they are related, i.e. what physical processes are behind their relation. Therefore, the physics of each of the phenomena in the chain is important and a deeper insight in this should help determining the boundary conditions of numerical simulations. We think that we are still in the era of specific case studies leading to geo-effectiveness or not, trying to really understand why a chain of events starting from a flare-CME with all the favorable conditions does or does not end with a geoeffective event. Only a few rare studies are conducted to explain non-geo-effective events (Mays et al., 2015; Thalmann et al., 2015). As already mentioned, statistical approaches are important to detect associations and correlations in the Sun-Earth chain of events. However, they are often biased by the choices of (only) geo-effective events and the related solar events. Only a few works concern a not biased sample due to a careful selection of events. For example, Bocchialini et al. (2018) studied all the sudden storm commencements (SSCs) events occurring during one year without suppressing any event and following the chain of events from the Earth to the Sun. It was particularly difficult to relate the SSCs occurring at L1 to corresponding solar events and also to find the solar sources of the CMEs involved.

In the present paper we follow a new approach, not biased by the pre-selection of the flares, and we consider all the X-class flares occurring in 2002 and we trace the full chain of events from the Sun to the Earth.

The paper is organized as follows. In Section 2 we first describe the catalogs from which we extracted the data that we used and then we address the methodology for following the chain of events from the Sun to the Earth. The geo-effectiveness is discussed based on four different measurements: the Dst minimum, SPE flux values, the Auroral Electrojet (AE) maximum values, and SSCs. In Section 3, we summarize the associations that we found between these phenomena (Table 1). Then we discuss the relationship between the characteristics of the flares, CMEs, ICMEs, and SPE events. In Section 4, we present in more detail the flare-CME chains of a few cases according to the longitude of their sources and particularly those for which all the parameters are favorable for being associated with geo-effectiveness. We try to find the answer to why the geo-effectiveness rate was so unusually low in 2002 (Gopalswamy et al., 2007). This study should give insight to enable narrowing the space of parameters and ultimately lead to improved forecasts.

\section{Data and Methodology}

\subsection{Data}

The recent statistical study of Bocchialini et al. (2018) was dedicated to the chain of events from the Earth to the Sun by starting their research considering all the registered SSCs in 2002. SSCs involve a sudden growth of the magnetic field strength at the Earth surface. Furthermore, SSCs related to ICMEs are commonly interpreted as being due to the shock preceding the ICME sheath. For 2002, it was found that the 36 SSCs that occurred were related to moderate $\left.\left(-100 \mathrm{nT}<\mathrm{Dst}_{\min }\right)<-50 \mathrm{nT}\right)$ or weak geomagnetic storms. On the other end of the chain, the SSCs were related to solar sources and only four of them correspond to a X-ray flare, i.e. about $30 \%$ (four among twelve). We here revisit the data of 2002 with a different approach in which we consider all the Xclass flares observed during one year (2002) listed in Table 1 and to follow the chain of 
events from the Sun to the Earth (until the magnetosphere). In the year 2002, at the end of the solar maximum period of solar cycle 23, 2727 flares (12 X-class, 219 M-class, and 2496 C-class flares) were registered by the GOES X-ray detector and listed by NOAA including their source region with their coordinates (ftp://ftp.swpc.noaa.gov/pub/warehouse/2002).

In a first step, we are looking for the CMEs that could be related to those $12 \mathrm{X}$ class flares in the CDAW/LASCO CME online catalog (http://cdaw.gsfc.nasa.gov/CME_list/index.html) (Yashiro et al., 2004). Those CMEs of which the first appearance time was close to those of the $12 \mathrm{X}$-class flares from the CDAW list were selected. To check the association with the flares, we carefully compare the SOHO/LASCO images with SOHO/EIT running difference images (see an example in Figure 1). We determine EUV features such as coronal dimming, post eruption arcades or erupting filaments. By determining whether the CME position and EUV features have spatial and temporal closeness, we select the related CMEs for each flare.

Then, we consider the solar energetic particles (electrons, protons, ions), mainly the solar proton events (SPE) listed in 2002 by the NOAA/Space Weather Prediction Center (NOAA/SWPC) (https://umbra.nascom.nasa.gov/SPE/). In this study SPEs are defined as an event where the number of incoming energetic $(>10 \mathrm{MeV})$ protons (Ip flux) exceeds 10 particles flux unit (pfu: proton number per $\mathrm{cm}^{2} / \mathrm{sr} / \mathrm{s}$ ) at geosynchronous satellite altitudes. We note that for some cases in the GOES curves in the low frequency range $((>10 \mathrm{MeV})$ there is a second maximum, higher than the first one which does not exist in the higher frequency bands. The second maximum is interpreted as an energetic storm particle event (ESP). The particles are trapped in the ICME shock and are locally accelerated in the vicinity of the Earth (Mäkelä et al., 2011). Therefore we consider only the time of the first maximum as the peak time of SPE which does not always correspond to the peak time found in the catalogs as we will show with one example in Section 3. Five SPEs are related to the X-class flares, among the 19 SPEs listed in the data base of solar energetic protons.

We follow the events at L1 by using the OMNIWeb service (http://omniweb.gsfc.nasa.gov/) and the AMDA service (http://amda.cdpp.eu) to draw the records of all the parameters (IMF magnitude, three IMF components, IMF inclination, Solar Wind plasma density, bulk velocity, radial temperature, $\alpha$-particle to proton ratio). Statistical surveys of geomagnetic storms and their interplanetary causes become more routinely accessible during solar cycles 23 and 24 due to the reliability of in-situ measurements in the interplanetary medium made by the two satellites WIND with the Solar Wind Experiments (SWE, Lepping et al. (1995)) and the Advanced Composition Explorer (ACE, Chiu et al. (1998); Stone et al. (1998)) with the magnetic field experiment (MAG) and plasma particle experiment (SWEPAM, (McComas et al., 1998; Smith et al., 1998)). These satellites provide in situ measurements at L1 respectively on the interplanetary magnetic field strength and its components, the plasma velocity and density. Published catalogs (Jian et al., 2006; Richardson \& Cane, 2010; Nieves-Chinchilla et al., 2019) are considered for ICME identification. We adopt the method described in Bocchialini et al. (2018) to identify the ICMEs. When the ICME shock velocity in catalogs is lower than the CME LASCO velocity and also lower than the ballistic velocity (the constant radial velocity that would permit to travel from the last CME LASCO observation to L1 at the shock arrival time), we associate the ICME to the CME and we report ICME in Table 1. Long periods of intense southward IMF are known to be related to strong geo-effective events as day-side magnetic reconnection can occur. The peak value of $B_{z}<0$ or the mean $\left(B_{z}<0\right)$ can be misleading as these can be strongly negative even for very short $B_{z}<0$ periods. Moreover, the mean $\left(B_{z}\right)$ is close to 0 for events with equivalent periods of northward and southward IMF even if it contains periods of long intense southward IMF. To estimate the IMF contribution to the geo-effectiveness we thus choose $B_{z}^{*}$ as done in Bocchialini et al. (2018). $B_{z}^{*}$ is computed by the integration over time of the southward IMF $B_{z}$ component, divided by the total interval duration (including periods of northward IMF). For ICMEs, 
$B_{z}^{*}$ is computed between the leading shock time at L1 and the time of $\min (\mathrm{Dst})$. For the other events, $B_{z}^{*}$ is computed between the flare peak time plus one day and the time of $\min (\mathrm{Dst})$, and we report the value in brackets in Table $1 . B_{z}^{*}$ values lower than $-5 \mathrm{nT}$ are expected to be correlated to $\min ($ Dst).

Magnetic disturbances of the magnetosphere are studied from indices derived from measurements of station networks. Many different indices exist and correspond to different locations of the magnetometers (see Menvielle, Iyemori, Marchaudon, and Nose (2011) for a detailed review). Here we chose two indices, viz. the auroral electrojet (AE) for stations located in the auroral region, and the Dst index for stations in low latitudes, and a list of rapid magnetic variations (SSC, storm sudden commencements) published by the Ebre Observatory. SSC events are usually related to quick magnetosphere compressions. Moderate auroral activity is achieved for AE $>1000 \mathrm{nT}$. AE values above $1500 \mathrm{nT}$ are related to an intense activity. For the Dst we use the definition of Echer, Gonzalez, Tsurutani, and Gonzalez (2008) and Gonzalez et al. (1994) to classify the geomagnetic storms as intense storm (Dst min $<-100 \mathrm{nT}$ ), moderate storm $(-100 \mathrm{nT}$ $<$ Dst $\left._{\min }<-50 \mathrm{nT}\right)$, and weak storm $\left(-50<\mathrm{Dst}_{\min }\right)$. We report in Table 1 the maximum of $\mathrm{AE}$ and the minimum of Dst observed for each event. For ICME events we pick up Dst ${ }_{\text {min }}$ and $\max (\mathrm{AE})$ values in the time interval starting from the leading shock time until the end of the magnetic ejecta observation time given in the previously mentioned catalogs. Other events (non-ICMEs) are a kind of reference sample. ICME geo-effectiveness can be estimated by comparing index values between ICME events and non-ICME events. We have to select index values of non-ICME events during an arbitrary period of time as there is by definition no ICME arrival time for these events. For the non-ICME events we consider a four day duration window starting from the flare peak time plus one day and we report the value in brackets. The time interval definition explains the small differences between our values and the one presented in Bocchialini et al. (2018). 


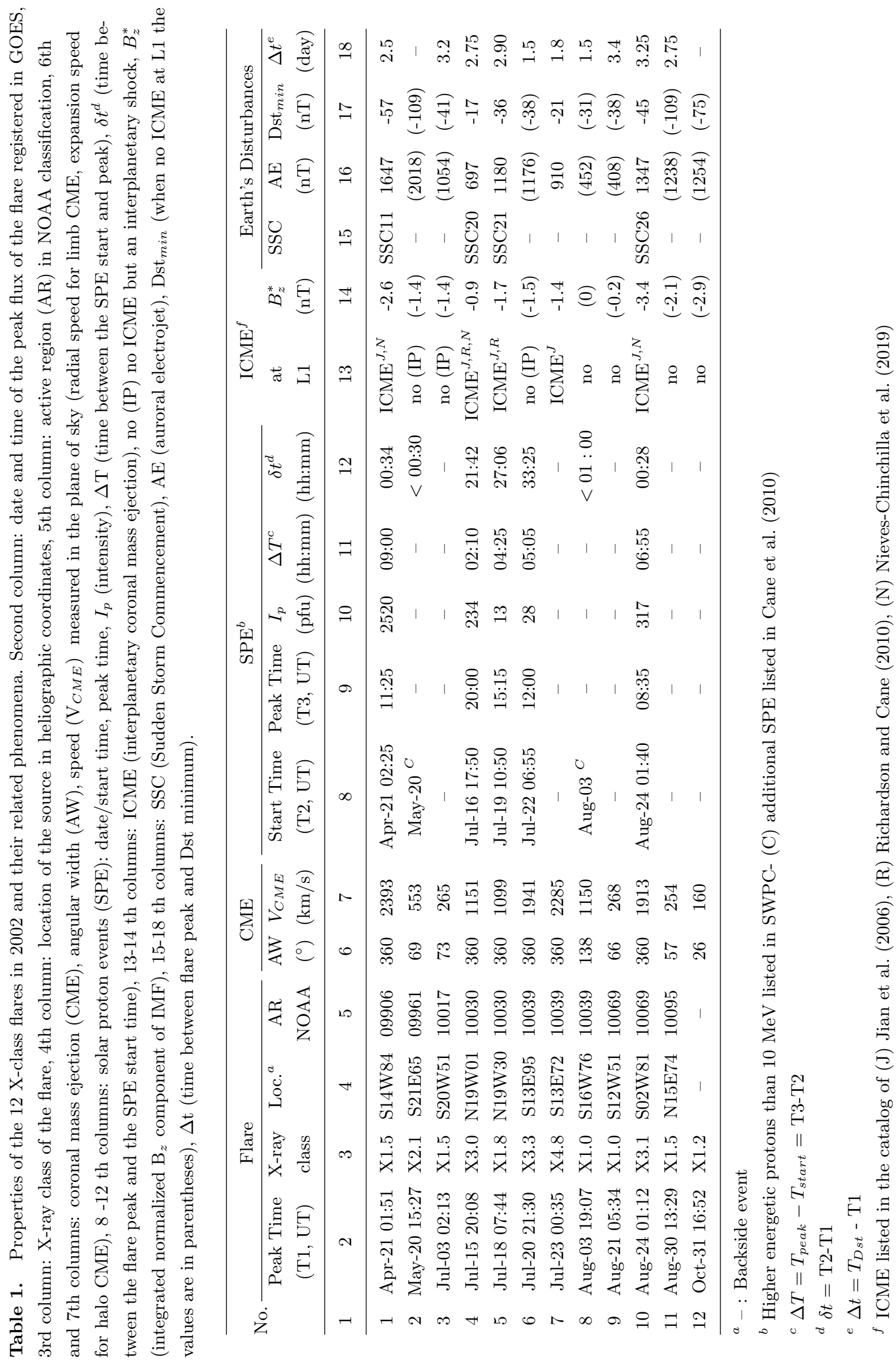




\subsection{Methodology for one example - Flare \#10 related effects}

The methodology is as following. For example we consider the X3.1 flare occurring on August 24 in the NOAA AR10069 located S02W81 (flare \#10 in Table 1). The source region was identified by the brightest region visible on the west limb in EIT images in $195 \AA$ coinciding in time with the onset of a high speed $(1913 \mathrm{~km} / \mathrm{s})$ halo CME listed in the CDAW catalog as shown in Figure 1. The flare erupted when the region was already close to the limb. As we have no possibility to get magnetic information of sources at the limb, we look at the region a few days earlier, namely on August 18. The region consists of a large sunspots group with a $\delta$ configuration (opposite following polarity surrounding the main leading polarity - see Figure 2 bottom panels). This magnetic complexity favors instability and magnetic reconnection leading to eruptions (Aulanier et al., 2010).
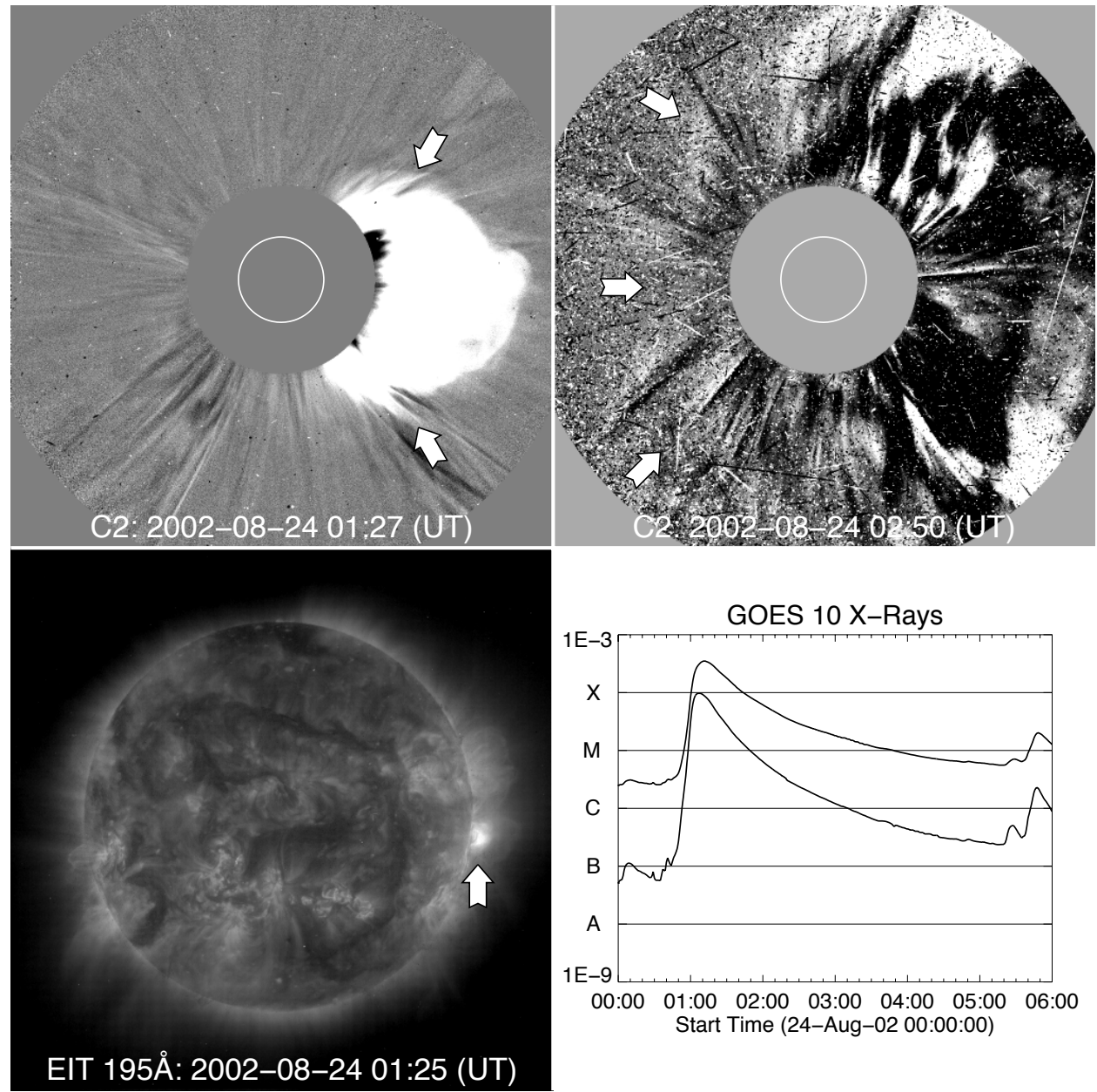

Figure 1. Example of the halo CME source at the West limb on August 242002 (flare \#10). Top panels: left, halo CME at 01:27 UT observed by the LASCO/C2 coronograph, right, difference images of the halo CME at 02:50 UT. The three white arrows indicate the front of the shock which appears even at the East limb completing the halo. Bottom panels: left, EIT image in 195 $\AA$ showing the bright flare at the limb in AR 10069 with an white arrow), right, GOES X-rays record during the flare. 

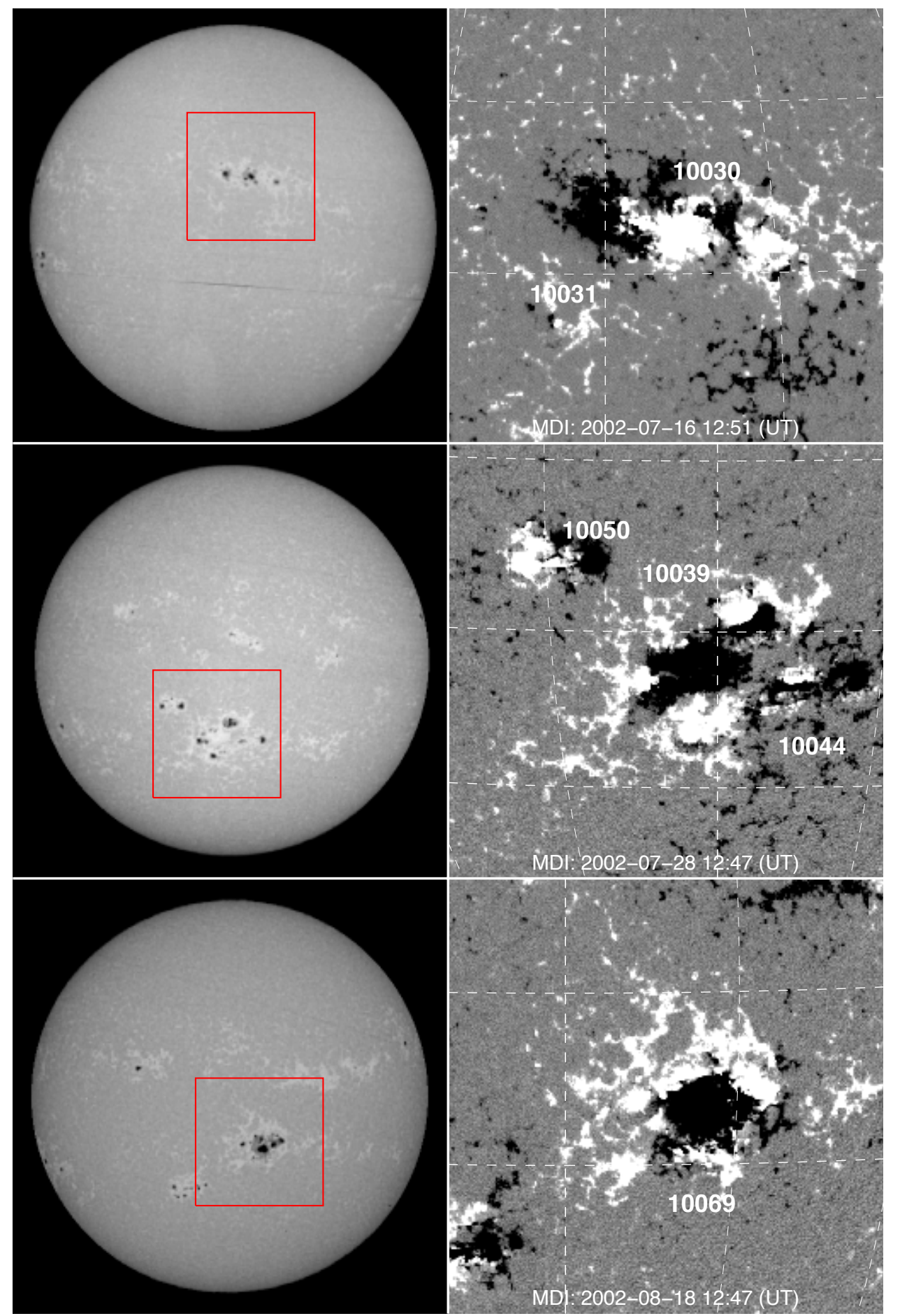

Figure 2. Active regions (ARs) crossing the central meridian in July and August before or after flare occurrence, (left column: Meudon spectroheliograms in Ca K1v line), and their complex magnetic field, the field of view corresponds to the red box on the left, (right column: MDI magnetograms from SOHO): from top to bottom - AR 10030 on 16 July between flares \#4 on July 15 and flare \#5 on July 18, - AR 10039 on 28 July between flares \#6 on July 20, \#7 on July 23 and \#8 on August 3, - AR 10069 on 18 August before flares \#9 on August 21, and flare \#10 on August 24 (see Table 1). 
Then we look at the Wind spacecraft data in a time window of four days after the CME launch. Solar wind and IMF quantities are plotted between August 24 and 28 in Figure 3. Following the evolution of the value of the $z$-component of the magnetic field at L1 (orthogonal component to the ecliptic plane) allows us to understand the possible reconnection in the IMF. $B_{z}<0$ is a favorable condition for having reconnection between ICMEs and IMF (Tsurutani et al., 1988).

Two successive discontinuities are observed in the IMF magnitude (top panel of Figure 3) at 26 August at 10:40 UT and at 14:00 UT. The first discontinuity is concomitant to the solar wind kinetic pressure increase seen on both the solar wind density (fourth panel from the top) and velocity (fifth panel). The second IMF intensification is related to the end of the density pulse. It is worth noting that the IMF is strongly southward (second and third panels) during several hours following the second discontinuity. ICMEs are reported during that period by Jian et al. (2006) (ICME1) and Nieves-Chinchilla et al. (2019) (ICME2). The magnetic obstacles identified in these studies are delimited by black arrows in top panel of Figure 3. A short magnetic ejecta is observed in ICME2 on the 27 August between 03:00 and 10:00 UT (low level of magnetic fluctuations and IMF coherent rotation; see second panel). This ejecta could be a short duration ICME with a positive helicity merging with the first one. In Figure 3 we indicate by a vertical dotted line the Dst min corresponding to the ICMEs. We choose the time of the second Dst minimum because the ICMEs are still observed at L1 at that time and thus the Dst activity is probably ICME-related (see Table 1).

Furthermore, with the C2 coronagraph of LASCO, a partial CME is seen around 20:50 UT on 23 August with a velocity of $860 \mathrm{~km} / \mathrm{s}$, from very close parts in the AR before the launch of the halo CME. This partial CME is caught by the halo CME. The limb location of the halo CME source can explain the poor aspect of the ICME observed at L1. The halo CME would correspond to the ICME between 27 August 03:00 to 10:00 UT according to the ballistic velocity analysis.

The ballistic velocity $v_{\text {bal }}(\approx 650 \mathrm{~km} / \mathrm{s}$ for this event) is the constant velocity that would permit an ICME to propagate from the solar corona at $t_{\odot}$ to L1 at the shock arrival time $t_{L 1}$. The shock seen at L1 arrives with a velocity of $\approx 430 \mathrm{~km} / \mathrm{s}\left(v_{L 1}\right)$ and matches the velocity criterion $\left(v_{b a l}>v_{L 1}\right)$. As the CME velocity $(1913 \mathrm{~km} / \mathrm{s})$ is also larger than the ballistic velocity, we can associate the CME related to flare \#10 to the observed ICME We thus tag this event ICME in Table 1.

The density pulse obviously results in the onset of a magnetic storm. The Dst then reaches a minimum: $-45 \mathrm{nT}$ between 19:00 and 20:00 UT on 26 August and again $-45 \mathrm{nT}$ on 27 August at 03:00 UT corresponding to the two different events. $B_{z}$ is large and northward at the beginning (26 August at 11:00 UT). It turns quickly strongly southward at 15:00 UT but continues with strong rapid fluctuations until the end of the second ejecta (ICME). It is consistent with the two successive minima of the Dst around $-45 \mathrm{nT}$.

We compute $B_{z}^{*}(-3.4 \mathrm{nT})$ between the shock arrival time at L1 and the last Dst minimum (blue dot line, bottom panel of Figure 3). Bocchialini et al. (2018) noted that values above $-5 \mathrm{nT}$ leads to moderate storms. Therefore, even if $B_{z}$ is strongly negative at the shock arrival time $(<-10 \mathrm{nT})$, if it does not last long enough (more than during half of the ICME duration) it does not cause a strong Dst decrease. The weak intensity of the storm is thus explained by the sign of $B_{z}$, i.e. the orientation of the IMF: the ICME magnetic field orientation at L1 is not optimum for causing large storm. We can also note a moderate auroral activity $\left(\mathrm{AE}_{\max }=1347 \mathrm{nT}\right)$ when the ICME is passing through L1.

Looking at the SPE data listed in 2002 by NOAA/Space Weather Prediction Center and the GOES curves we determine approximately the SPE start time (T2) and the SPE peak time (T3) avoiding the ESP (Energetic Storm Particles) events (Figure 4). Their 


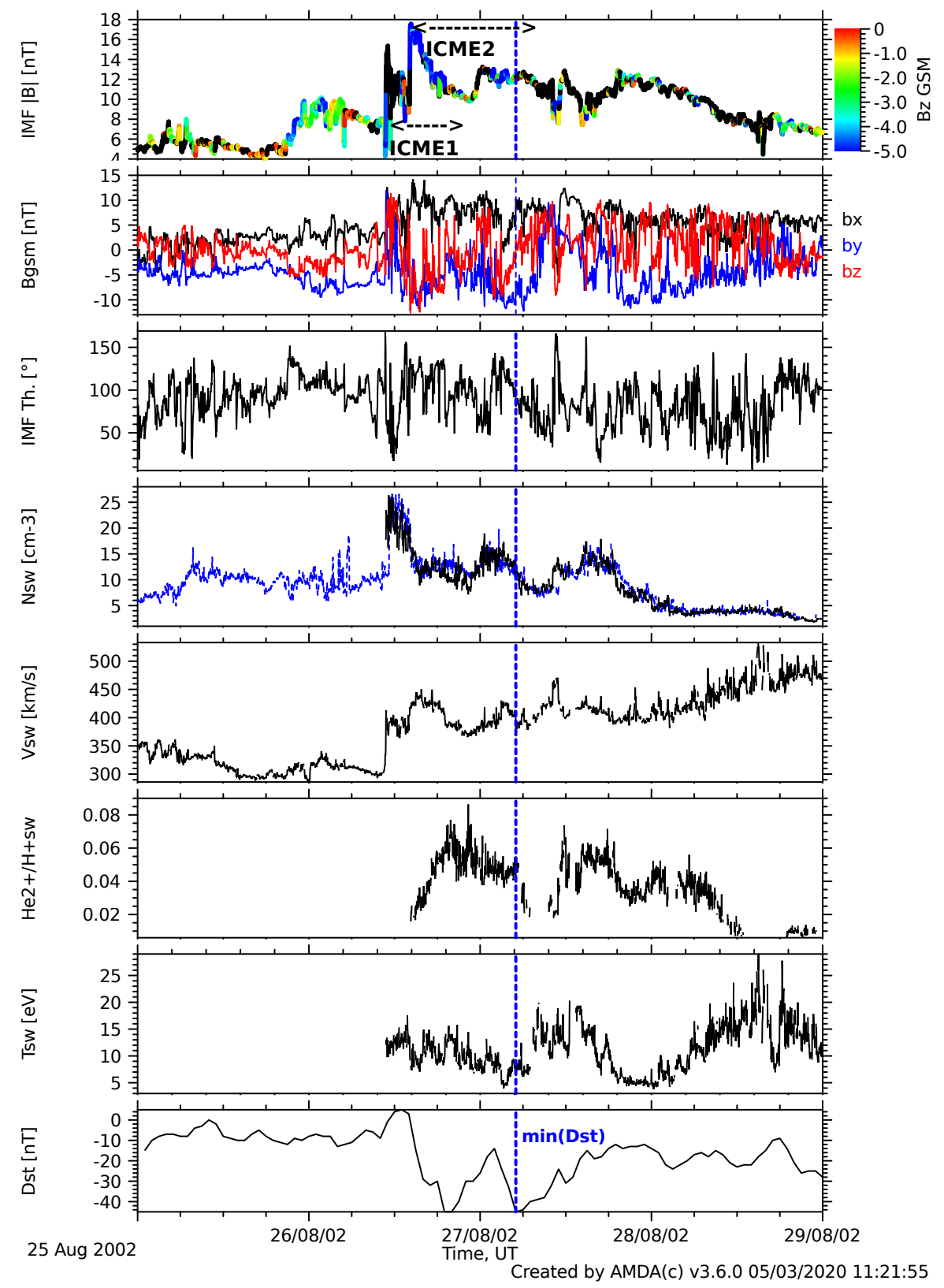

Figure 3. L1 observations (ACE data) starting from 24/08/2002 00:49 (detection of flare \#10) and during five days. IMF (Interplanetary Magnetic Field) magnitude, three IMF components and IMF deviation angle from $z_{G S M}$-axis are plotted in the three top panels (ACE/MAG data). The solar wind plasma density, flow velocity, $\alpha$-particle to proton ratio, and temperature are plotted in the next four panels (ACE/SWEPAM data). As there are some gaps in ACE data, density values from WIND/SWE instrument are plotted in blue. In the bottom panel, Dst ground-based index is plotted and the blue vertical dashed line indicates the Dst minimum related to flare/CME \#10. ICME1 and ICME2 are the ICMEs proposed by Jian et al. (2006) and Nieves-Chinchilla et al. (2019) respectively. The time interval of each ICME is indicated in the top panel. 
difference $(\delta \mathrm{t})$ could bring an information about the location of the acceleration process. Flare/CME \#10 is related to an SPE with a short $\delta \mathrm{t}$ (28 minutes) after the flare peak time (T1), which means that the energetic particles could come directly from the flare reconnection site. However the $\mathrm{CME}$ is concomitant with the flare and has a very fast speed (more than $2000 \mathrm{~km} / \mathrm{s}$ ). So it is difficult to disentangle if the SPEs come from the flare and/or the CME/shock contributions (Cane et al., 2010). SPEs are visible in the three frequency bands even in the more energetic one (Ip > $100 \mathrm{MeV}$ ). Such an event occurring in the central meridian would have been seriously geo-effective.

In summary, the flare-CME pair \#10 which has a solar source near the West limb (W81) is related, nevertheless, to an ICME at L1, to a non negligible $\operatorname{Dst}_{\min }(-45 \mathrm{nT})$ and a SPE in the Sun-Earth chain. The tentative analysis of this chain of events shows the difficulty to relate one event with another one even if the times are consistent with the propagation velocities. We show that the conditions of the IMF and the passage of previous CMEs are important to interpret this event.

\section{Characteristics of the $12 \mathrm{X}$-class flares and the chain of related events}

\subsection{X-class flares and corresponding CMEs}

The twelve X-class flare-CME pairs and the chain of events from the Sun to the Earth according to the methodology described above are listed in Table 1 . The first five columns indicate the characteristics of the flares: number in the list, peak time, X-rays class, the coordinates of the solar source and the NOAA AR number. The X-class flares are all originated in active regions with large sunspot areas and complex magnetic structures mostly due to new emergence flux in the middle of the ARs (Figure 2). The flare

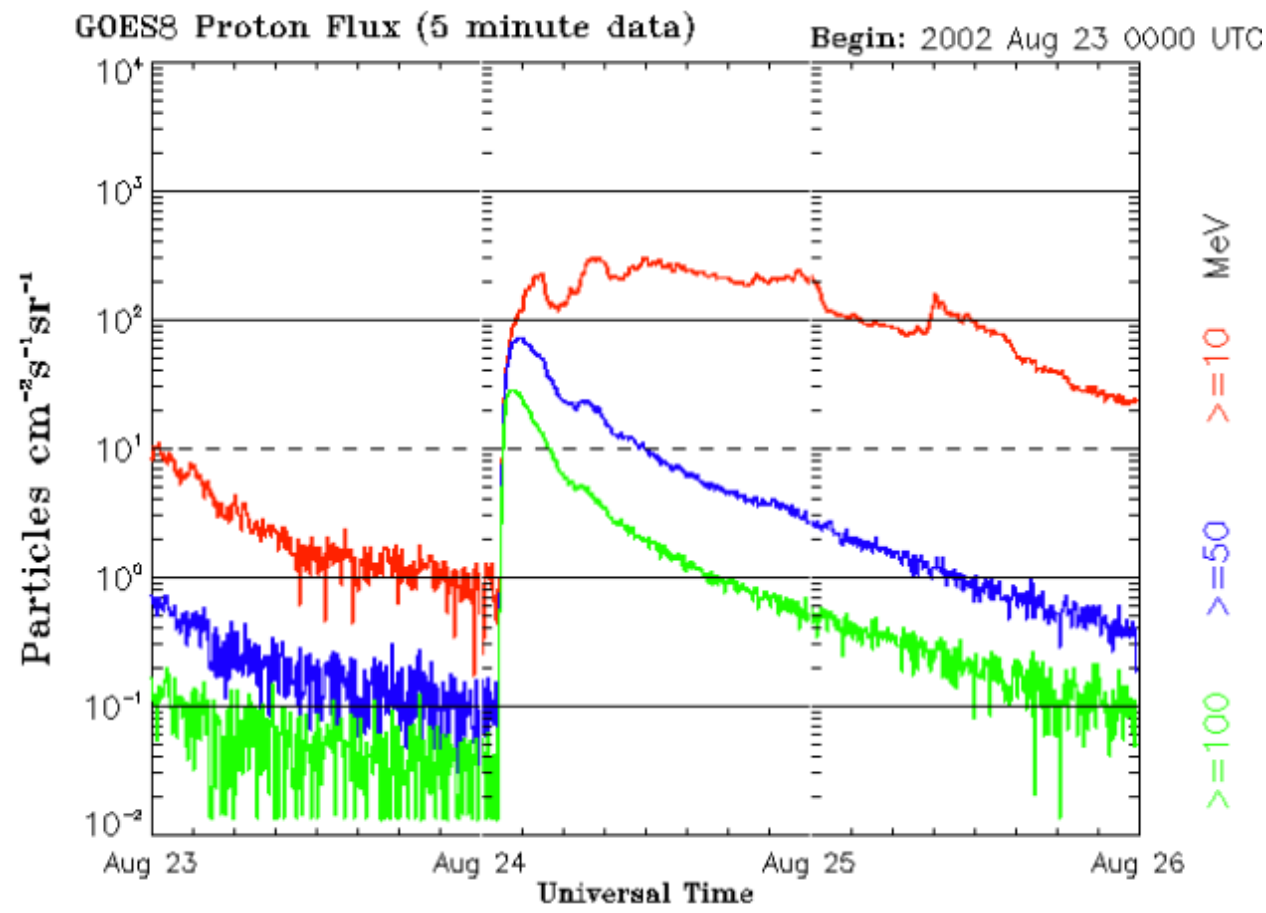

Figure 4. Variation of SPE on August 24 related to flare \#10. In the low energy channel (red curve) several maxima occur during the gradual phase that are called Energetic Storm Particles $(\mathrm{ESP})$. 

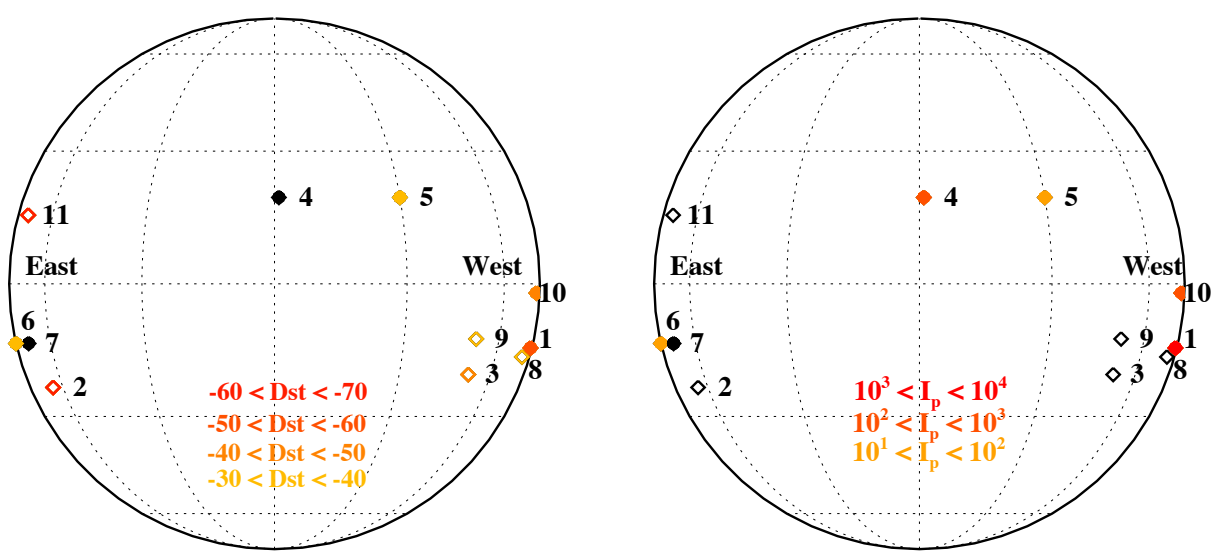

Figure 5. Left panel: longitudinal distribution of geomagnetic storm events associated with 12 X-class flares on 2002. The red- orange- yellow diamonds indicate the flare sources associated with $-30 \mathrm{nT}<$ Dst $<-60 \mathrm{nT}$. The full diamonds are associated with full halo CMEs in both panels. Right panel: Longitudinal distribution of the SPEs associated with X-class flares on 2002 (red- orange- yellow diamonds for $10^{1} \mathrm{pfu}<\mathrm{I}_{p}<10^{3} \mathrm{pfu}$ ). The black diamonds are the location of the flare sources not associated to any SPE.

occurrences are mainly clustered in one time period from 15 July to 30 August. During this time large sunspot areas consist of $\beta, \gamma, \delta$ spots (in the Hale nomenclature) gathered in one or two ARs.

Indeed, large sunspot areas with complex magnetic field are favored for X-class flares (Aulanier et al., 2013; Schmieder, 2018). It is in agreement with the good relationship found between the size of sunspots and the intensity of flares (Sammis et al., 2000). The source of the $12 \mathrm{X}$-class flares listed in the fourth column of Table 1 are located mainly close to the limb (see Figure 5). Moreover, two flares (flare \#6 and flare \#12) have a backside solar source. However, the source of flare \#6 could be identified by bright loops prominent over the limb before the AR 10039 passes on the disk (Figure 2). Therefore, the flare source is defined as S13 E95 in the column 4 in Table 1.

The 6th and 7th column concern the CME characteristics: width and velocity. We note that all the X-class flares are associated with a CME: half of them with a halo CME with a velocity between $1000 \mathrm{~km} / \mathrm{s}$ and $2000 \mathrm{~km} / \mathrm{s}$. In this context we will consider the $\mathrm{X}$-class flare and its associated CME as a global event, in our study named a flare-CME pair. The flare and CME relationship is shown in Figure 6. The flare flux is relatively well correlated to the CME speed and with the CME width as well. This confirms previous, more extensive statistical studies associating flare flux with CMEs (Hundhausen, 1997; Moon et al., 2002; Yashiro et al., 2006; Gopalswamy et al., 2009). We are in agreement with the identification of Yashiro et al. (2004), except for one flare (flare \#12 in our flare list), which was considered as a CME-less flare (Klein et al., 2010). Finally, all the $12 \mathrm{X}$-class flares are associated with at least one CME (see Table 1).

\subsection{Mass and kinetic energy of CME}

The intensities in coronagraph images result from Thomson-scattered light by coronal electrons (e.g., Minnaert (1930)), and thus the number of electrons can be obtained from the observations by considering the Thomson scattering theory (e.g., Billings (1966)) While the mass estimation also depends on the 3D geometry of the source (e.g., Vourlidas 
and Howard (2006)), the CME mass has been determined under the assumption that all mass lies on the plane-of-the-sky (Vourlidas et al., 2000). After the multiple viewing perspective observations became available by STEREO in late 2006, there have been attempts to correct the CME mass with the 3D geometry (Colaninno \& Vourlidas, 2009; Carley et al., 2012; Feng et al., 2013). Due to the absence of the STEREO observations in our study, the CME mass has been derived under the plane-of-the-sky assumption.

We use the LASCO C2 images subtracted from a pre-event image to remove the background emission including the noise, and the residual was used for the calculation of the CME mass. The mass was derived from all images taken at times when the CME body passes through the height range from 2 to 6 solar radii, and the maximum values are listed in Table 2. To calculate the mass from C2 images, we used an IDL procedure, 'calc_cme_mass.pro' that can be found from the Solar Software. Note that the mass varies with time because of the propagation and the expansion as they are passing through the image plane, and the CME bodies became larger than the C2 FOV. In this regard, the calculated mass is not the total mass of the whole CME body, but the maximum value could be a proxy of it owing to conservation of mass. Once the mass is determined, the kinetic energy can be computed with the CME speed listed in Table 1. We note that all the halo CMEs have a kinetic energy larger than $10^{32}$ ergs except the CMEs \#4 and

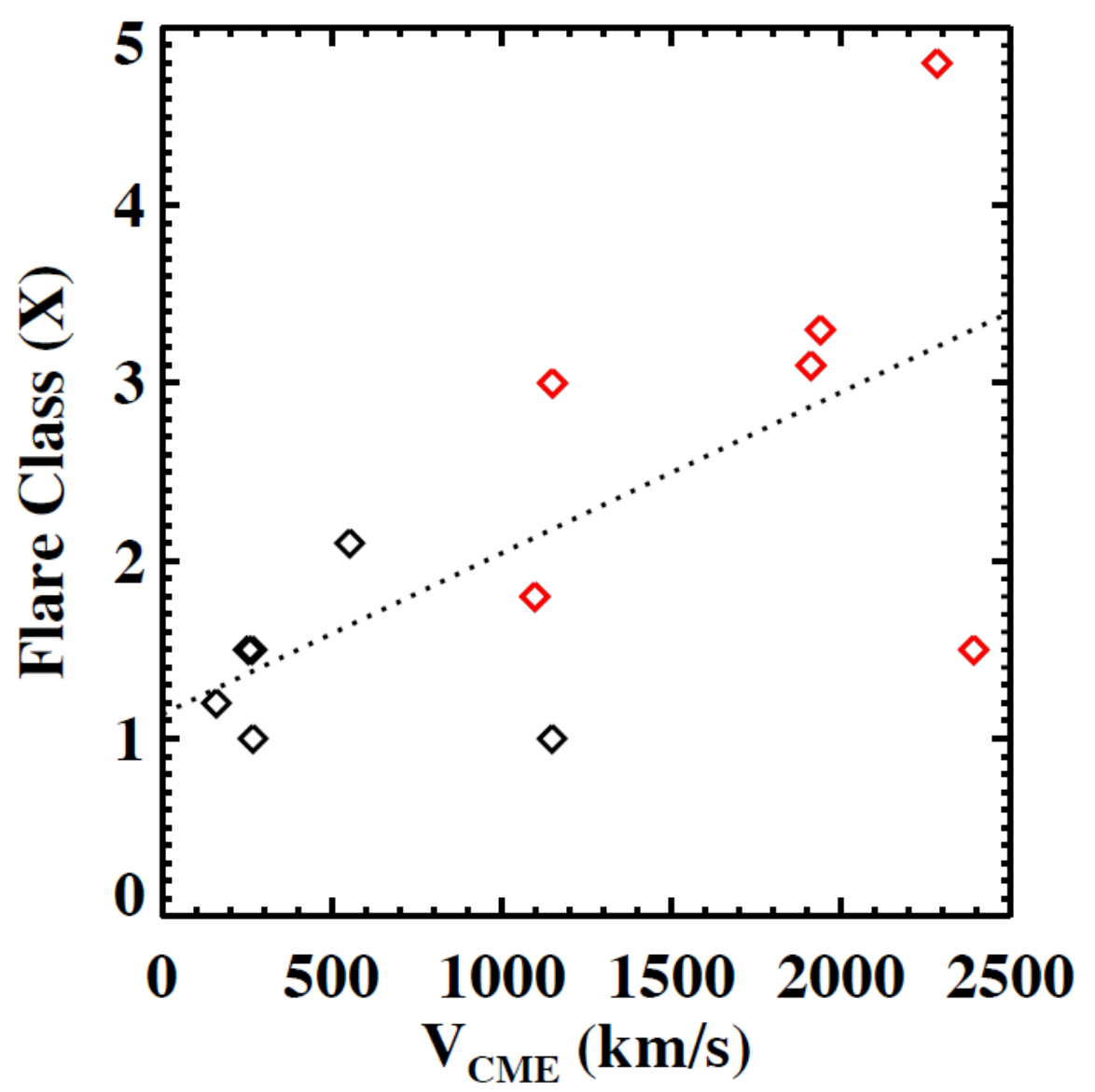

Figure 6. Relationship of the $12 \mathrm{X}$ class flares and CMEs in 2002. $\mathrm{V}_{C M E}$ is the speed of the CME (see Table 1). The red diamonds correspond to halo CMEs, the black diamonds correspond to non halo CMEs. 
Table 2. Characteristics of the CMEs, halo and partial related to the X-flares

\begin{tabular}{c|l|l|l|l}
$\begin{array}{c}\text { Number } \\
\text { flare }\end{array}$ & $\begin{array}{l}\text { time of CME } \\
\text { UT }\end{array}$ & CME shape & $\begin{array}{l}\text { mass of CME } \\
{[\mathrm{g}]}\end{array}$ & $\begin{array}{l}\text { kinetic energy } \\
{[\mathrm{ergs}]}\end{array}$ \\
\hline 1 & 21 Apr. 01:27 & halo & $1 \times 10^{16}$ & $3 \times 10^{32}$ \\
2 & 20 May 15:25 & & $3 \times 10^{15}$ & $5 \times 10^{30}$ \\
3 & 03 July 02:10 & & $7 \times 10^{14}$ & $3 \times 10^{29}$ \\
4 & 15 July 21:30 & halo & $6 \times 10^{15}$ & $4 \times 10^{31}$ \\
5 & 18 July 07:49 & halo & $1 \times 10^{15}$ & $9 \times 10^{30}$ \\
6 & 20 July 21:30 & halo & $9 \times 10^{15}$ & $2 \times 10^{32}$ \\
7 & 22 July 18:30 & halo & $5 \times 10^{15}$ & $1 \times 10^{32}$ \\
8 & 03 Aug. 18:59 & & $2 \times 10^{15}$ & $1 \times 10^{31}$ \\
9 & 21 Aug. 05:28 & & $2 \times 10^{15}$ & $9 \times 10^{29}$ \\
10 & 24 Aug. 00:49 & halo & $2 \times 10^{16}$ & $3 \times 10^{32}$ \\
11 & 30 Aug. 12:47 & & $5 \times 10^{14}$ & $1 \times 10^{29}$ \\
12 & 31 Oct. 16:47 & & $7 \times 10^{14}$ & $9 \times 10^{28}$
\end{tabular}

\#5, which have a slight lower energy around $10^{31}$ ergs. The non halo CMEs have a kinetic energy, one to three orders lower than the halo CMEs.

The CME kinetic energy is a lower limit as the real CME velocity could be larger than the apparent one, due to projection effects.

\subsection{Solar energetic particles}

Columns 8 through 12 of Table 1 concern the SPE characteristics: start date and time, peak time, intensity, $\Delta \mathrm{T}$ (time between the start of the SPE and the peak), and $\delta \mathrm{t}$ (duration time between flare peak and start of the SPE). $\Delta \mathrm{T}$ gives an estimate of the duration of the rising phase of the phenomena (between 2 to $9 \mathrm{~h}$ ). $\delta$ t denotes the traveling time of the ICME before acceleration of particles. These are all approximate values and concern the low frequency band of energetic particles $(>10 \mathrm{MeV})$. Only five flare - CME pairs are related to SPEs. In Table 1 we list the flux of these five SPE events in pfu. We identify a new SPE event not listed in the NOAA/SWPC data base: it concerns flare \#6 (see Table 1). The pfu values are weak for most of them but there are not negligible with a highest value of $2520 \mathrm{pfu}$ for the flare/CME \#1. Indeed, commonly only one SPE event with a flux $>800$ pfu occurs per year (Klein et al., 2011). The maximum value that has been measured ever was $40000 \mathrm{pfu}(24 / 03 / 1991)$ during the solar cycle 22 and 6300 pfu $(07 / 03 / 2012)$ during the solar cycle 24.

Cane et al. (2010) found 40 SPEs in 2002 with six of them related to an X-class flare. Four of them are listed in the SWPC catalogue and two of them (flares \#2 and \#8 - Table 1) are not. The definition of SPE events in Cane's paper is based on the intensity of protons in the domain 25 to $30 \mathrm{Mev}$. However, it is difficult to identify an enhancement of the flux in the low energy band around $10 \mathrm{Mev}$ (GOES curves). The Ip is too small to be listed in the SWPC catalogue. Therefore, we do not use these two events in the following discussion. However, we find one more SPE event related to flare \#5. The occurrence of a large type III, seen in WIND confirms our identification as an impulsive event. Long duration events or gradual events indicate that energetic particles are accelerated during a long period of time in the heliosphere, corresponding to the travelling time of the ICME toward the magnetosphere (Tsurutani et al., 2009). There is a slight correlation between CME speed and $\delta$ t and with the SPE flux (Figure 7). Short $\delta$ t corresponds to fast CMEs. SPEs are often associated with the local acceleration of the particles by interplanetary shocks. 

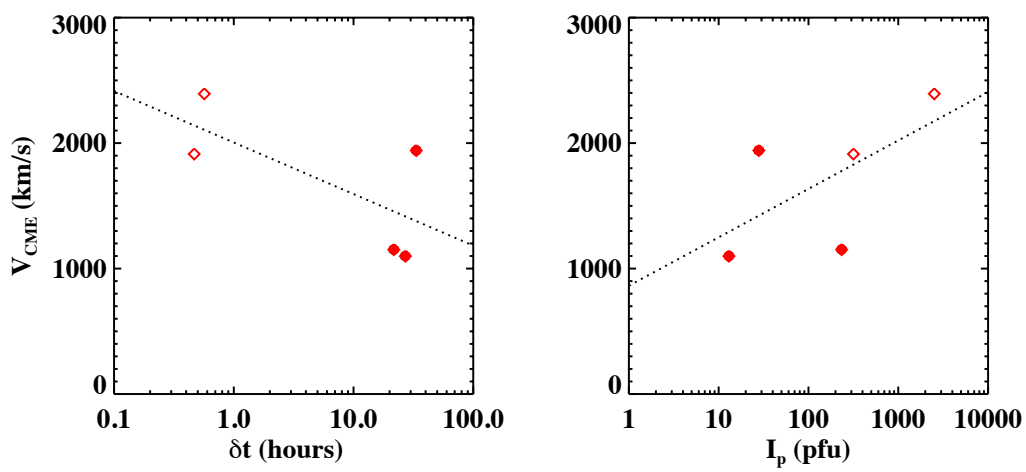

Figure 7. Relationship of X-class flare-elated CME speed and solar proton events (SPE) in 2002. The energy Ip is in pfu, $\delta \mathrm{T}$ is the time difference between the flare peak and the SPE start times. The full/empty red diamonds correspond to long/short SPE-flare delays.

\subsection{ICMEs' arrival and geomagnetic indices}

Columns 13-14 of Table 1 gather the L1 observations related to each flare-CME pair. Three categories of events are listed in column13: "ICME" stands for clear ICME signature related to halo CME, "no" stands for absence of ICME signature, and "no (IP)" stands for no ICME but with the detection of an interplanetary shock (IP) signature probably not related to the halo CME. We could identify unambiguously five ICMEs (\#1, $\# 4, \# 5$, \#7, and \#10), all in agreement with previous catalogs (Jian et al., 2006; NievesChinchilla et al., 2019; Richardson \& Cane, 2010), three "no (IP) cases (\#2, \#3, and \#6) with a velocity at L1 lower than the ballistic velocity and four "no" cases (\#8, \#9, \#11 and \#12), with no time-consistent association when performing the velocity comparison (CME velocity, solar wind velocity, and ballistic velocity). Among these four cases of flare-CME pairs the associated CMEs are slow and they are not observed at distance larger than $10 \mathrm{R}_{\odot}$.

The time is not in favour with the association with the CME because the ballistic velocity is lower than the velocity at the Sun and at L1. Finally in column 14, we indicate the southward IMF component $\left(\mathrm{B}_{z}<0\right)$ integrated and normalized value over time $\left(B_{z}^{*}\right)$. In Bocchialini et al. (2018) $B_{z}^{*}$ above $-5 \mathrm{nT}$ were associated with small Dst depression (low geo-effectivity). We can note that the minimum $B_{z}^{*}$ value is $-3.4 \mathrm{nT}$ (\#10) indicative that no ICME is expected to be strongly geo-effective.

Columns 15-18 in Table 1 indicate the Earth's disturbances: SSC, AE, Dst, and the delay $\Delta t$ between the peak flare time and the minimum of the Dst. We recap that Dst values in parenthesis in Table 1 are not attributed to the considered flare-CME pair. This illustrates the utility of performing a detailed association between the Earth and 
the Sun and not taking a fixed time window. The overall geo-effectiveness based on min(Dst) and $\max (\mathrm{AE})$ values consecutive to ICMEs is rather low. The four SSCs are associated with four X-rays flare-CME pairs having also a SPE signature (Table 1). They display an enhanced auroral activity shown by an important AE index. A fifth flare-CME pair (\#6) is also associated with SPEs. However, this pair is not associated with any SSC neither with a Dst minimum. This could be explained by the backside source location of the flare. In column 18 of Table 1 we see that the disturbances arrival time $\Delta t$ is between 1.5 and 3.4 days in the environment of the Earth. This leads to a mean value of the time window of 2.6 days.

In conclusion, the analysis of the Sun-Earth chain of all these events shows that there is not a significant relationship between Dst values and flare-CME characteristics (speed and width) (Table 1 and Figure 5). It is worth noting that none of the fast halo CMEs in our sample causes a strong Dst depression, despite they display the most relevant proxies for geo-effective event predictions. The number of our events is certainly too small to make a strong statistical conclusion. The source longitude is certainly a cause of this anomaly.

\subsection{Flare-CME source longitude and geo-effectiveness}

The longitudinal location of solar flare-CME source is commonly a good indicator to predict its geo-effectiveness. It is well known that limb events are less geo-effective than those originating around the central meridian (Webb, 2002; Richardson \& Cane, 2010; Cid et al., 2012; Lee et al., 2014). However, it is not 100\% reliable (Webb, 2002; Vasanth et al., 2015). In Cid et al. (2012), limb halo CMEs refer to a source longitude $>80$ degrees. We here adopt the same definition. We have already mentioned in section 3.1 the large proportion of our events having a source close to the limb (limb events) (Figure 5). It is an unusual high rate of limb sources in 2002 and it may explain the low geo-effectiveness of our sample.

We compare the longitudinal distribution of the source regions of our sample limited to the $12 \mathrm{X}$-class flare-CME events in 2002 to the longitudinal distribution of the source regions of the CMEs related to all the geomagnetic storms occurring in 2002 found in Bocchialini et al. (2018): the percentage of sources close to the limb is much higher for our sample $(7 / 12-58 \%)$ than the mean value $(10 / 26-28 \%)$ found in the sample of Bocchialini et al. (2018). Dst-based geo-effectiveness of all the $12 \mathrm{X}$-class flares are weak, with only one moderate storm (flare \#1) (Table 1, Figure 5). According to Gopalswamy et al. (2007) and Cid et al. (2012) such limb events (7 among 12 events) cannot lead to intense geomagnetic events, only moderate or weak events are expected. It is consistent with what we have observed.

SPE events depend also on the West-East longitude of the solar sources. Among the five SPE events that are listed in Table 1 four of them have a West source. Particles coming from a West source have stronger flux than those coming from the East due to the systematic eastward deflection of ICMEs when they interact with the Parker spiral interplanetary magnetic field (Parker, 1958; Zurbuchen \& Richardson, 2006). This is why the longitude of our SPE event sources corresponds mainly to the West side of the Sun (Figure 5).

Figure 5 summarizes the main results of the flare source longitude. For the ICME subset there is a relationship between the flare source longitude and Dst minimum values. However, the sample is too small to draw a robust conclusion. We note also a trend for $\mathrm{AE}$ to occur for West side sources. More sources of X-ray flare-CME pairs are located in the West part (70\% from the West part similar to the $61 \%$ found in Bocchialini et al. (2018)). This is also in agreement with the statistics studies of large number of events (Richardson \& Cane, 2010). From our (small) sample of 12 flare-CME pairs we have the following classification: 
- four sources are on the East side (flares $\# 2, \# 6, \# 7$, \#11), two are related to an ICME.

- five sources are on the West side (flares \#1,\#3, \#8, \#9, \#10), two are related to an ICME.

- two sources are front side (flares \#4, \#5) and are related to ICME.

- one source location is backside (flare \# 12).

Seven flare-CME pairs have no ICME signature at L1. None of them are in the disk central region: four of them $(\# 2, \# 6, \# 7, \# 11)$ have a solar source in the far East disk side, two of them $(\# 8, \# 9)$ in the far West side, and one (\#12) has a source on the backside. Moreover, six of these flare-CME pairs (all but \#6) are associated with narrow CMEs. The angular width of these CMEs (AW in the 6th column in Table 1) varies between 57 and 140 degrees. The longitudinal location of their solar sources and the narrow width of the CMEs could be good reasons why they have no signatures at L1. The CMEs pass far away from the Sun-Earth direction.

In conclusion, we note the the source longitude is an important parameter for the geo-effectiveness of the events (e.g. Dst, SPE). The flare-CME subset related to ICME or SPE intersects with the halo CMEs subset of our events. The limb source longitude of the CMEs can explain the poor geo-effectiveness measured in 2002, the ICMEs avoiding the Sun Earth axis. However, it does not explain the cases of the front-side CME source events for which the weak $B_{z}$ component of the IMF cannot be predicted (column 14 in Table 1). The full chain of the events related to the six X-class flare associated with halo CMEs is discussed in details according to their solar source longitude and the IMF configuration in the Annex.

\section{Discussion of the results}

\subsection{Summary of the study of the $12 \mathrm{X}$-class flares}

In the present paper we focus on the full set of the 12 X-class flares observed in 2002, their solar sources, the accompanying CMEs, and their related geo-effective events (indicated by the Dst index, the AE index, and the SPEs fluxes, Table 1). This study tries to answer a question that became pertinent after the paper of Bocchialini et al. (2018) in which only four X-flares among the twelve $2002 \mathrm{X}$-class flares have been related to geoeffectiveness defined through the Sudden Storm Commencements (SSCs). All the 12 Xclass flares analyzed here are associated with at least one CME, and six of them even with fast halo CMEs $(1000 \mathrm{~km} / \mathrm{s}<\mathrm{V}<2000 \mathrm{~km} / \mathrm{s})$, while the other six CMEs are characterized by a slow propagation speed and a narrow width CME. We follow the fast CMEs as they propagated through the heliosphere and search for their signatures at L1 in a reasonable time window ( 1 to 4 days after onset). We find that the average arrival time of the ICMEs and IPs is 2.6 days for our small sample. Five flare-CME pairs among the six with fast halo CMEs could be related to ICMEs producing geomagnetic storms, detected by a minimum in the Dst index.

We identify the solar sources of the X-rays flare-CME pairs and find an exceptionally high rate of sources occurring near the limb (more than 58\%). Statistically it is known that CME limb source is not a favorable location for producing intense geomagnetic storms (Gopalswamy et al., 2009; Cid et al., 2012). In our sample, all the Dst minima are very weak, and only one flare-CME (\#1) is related to a moderate geomagnetic storm (min Dst $<-50 \mathrm{nT}$ ). The flare duration seems to be an important parameter in the chain of flare to geo-effectiveness since we find a general trend for Dst, AE and Ip to be larger with longer duration flares.

Moreover, six cases among our sample display all most relevant proxies for geo-effective event predictions, e.g. fast halo CMEs. 
For the five of them followed by ICMEs, the shock arrival times observed at L1 are consistent with their ballistic (constant) velocity. Bocchialini et al. (2018) showed that this is a relevant parameter to test the ICME/CME association. Among this set of these six flare-CME pairs we identify four cases with a limb source. The associated ICMEs are identified among complex fluctuations of the magnetic field due to shocks and sheaths in front of the ICMEs leading to a small value of $\mathrm{B}_{z}^{*} \cdot B_{z}^{*}$ is computed by the integration over time of the IMF $B_{z}$ component, divided by the total interval duration. Then, even limb events could be detected at L1. The low B ${ }_{z}^{*}$ explains their low impact on the magnetosphere.

Considering the SPEs, in our sample of twelve X-rays flares, five flares (flares \#1, $\# 4, \# 5$, \#6, and \#10) are associated with an SPE event. They are also associated with an ICME. The percentage of X-class flares related to SPE events associated with an ICME is here thus $40 \%(5 / 12)$. In spite of our small sample, this is a similar percentage than the one found by Miteva et al. (2018) for the whole solar cycle 23. The corresponding sources of the SPEs are located at the West side or at disk center, regions which are effectively favorable for the propagation of accelerated particles. Only one X-class flare (namely flare \#1) among the twelve is accompanied by a large SPE flux (2520 pfu). For the SPEs, we confirm the conclusion of Klein et al. (2011): these authors found that during 10 years (1996-2006) among 69 GOES X-class flares only 10 SPE events (one per year) have a proton number larger than 800 pfu. Furthermore, we note that we could identify two more SPE events for 2002 not discussed in Klein et al. (2011), namely related to flares \#5 and \#6 which occurred in July. More generally, all the five SPEs found are related to X-class flares with halo CMEs and to SSCs (except flare \#6 with no relation to an SSC).

All the SPE delays between flare peaks and SPE start times, are in good agreement with Cane et al. (2010). The former authors interpreted the long delays by "a slow rise of the event". The large extension of the CMEs with spherical extended shock front could explain the long delay of accelerated energetic particles particularly when the source is close to the limb (Kwon \& Vourlidas, 2018). The two short delay cases (\#1, \#10) (less than one hour) could be interpreted as due to magnetic reconnection in the flare sites. Kim et al. (2015) confirmed that the largest SPE of 2002 (case \#1) is related to proton accelerated at the flare site. Case \#10 has very close onset times for flare and CME. Therefore, it is difficult to distinguish the SPEs coming from flare reconnection from those due to $\mathrm{CME}$ shocks.

However, the surprising cases are the two X-flare-halo CMEs related to flares \#4 and \#5 with their sources near the disk center: they are related to multiple interacting CMEs with one halo CME which is a favorable case of geo-effectivity (Lugaz et al., 2017). But the ICMEs associated with these flare-CME pairs have also a weak southward orientation of $\mathrm{B}_{z}^{*}$. More generally, low magnetic field strength enhancement indicates that the ICME should have been deflected in the interplanetary medium. This is consistent with the recent review of Manchester et al. (2017) who wrote about "all the different ways CMEs and ICMEs are rotated, reconfigured, deformed, deflected during their journey through the solar wind." The ICME-Earth geometry is an important parameter as demonstrated by Cho et al. (2017) after considering a single C-class flare leading to a large disturbance and a single X-class flare leading to weak geomagnetic effects. We conclude that for the two X-flare-halo CMEs (\#4, \#5), satisfying all the favorable proxies (source location, long flare duration, critical mass, large kinetic energy) to forecast an intense geo-effective storm is not a sufficient condition. This demonstrates also that energetic flares with fast front side halo CMEs never lead to strong geomagnetic disturbances in 2002. Other parameters have to be taken into account (e.g., the direction of B, the $\mathrm{B}_{z}^{*}$ value). 


\subsection{Forecast of SEP - Flares}

We showed that the $12 \mathrm{X}$-class flares were mainly related to three large complex active region areas which produced recurrent and long duration flares in July and August, a similar scenario that leads commonly to extreme Space Weather events (e.g. the Halloween events in 2003). Moreover, we need to understand the magnetic configuration of the solar sources and their stability for forecasting a geo-effective event before observing the CME (Török \& Kliem, 2005; Ishiguro \& Kusano, 2017). The magnetic topology of the active regions is a key parameter to understand if the flare will be followed by a halo CME which could be geo-effective (Schrijver, 2007; Y. Wang \& Zhang, 2007; Falconer et al., 2011; Zuccarello et al., 2017; Kontogiannis et al., 2019). It is crucial to determine if the flare will be eruptive and if particles can escape as demonstrated by Amari, Canou, Aly, Delyon, and Alauzet (2018). Large sunspot groups with a complex magnetic field such as in 2002 are not sufficient to predict geo-effective events (see Figure 2). For example if the magnetic field above the active region is too strong (e.g. a strong magnetic cage), the magnetic energy can only be released as thermal energy producing Xclass flares without CME and without related geo-effective events (SEP or Dst) (Mays et al., 2015; Thalmann et al., 2015).

Forecasts of energetic flares depend strongly on the amount of free (non-potential) magnetic field energy stored in an active region (Kim et al., 2017; Guennou et al., 2017). The free magnetic field energy depends on the twist and the entanglement of the magnetic field in an active region. The twist of the magnetic field lines corresponds to the magnetic helicity of an active region (Pariat et al., 2005; Demoulin et al., 2006; Dalmasse et al., 2015; Linan et al., 2018). In order to fully understand the chain from the Sun to the Earth, the partition of energy in the ARs should be evaluated. However it is difficult to forecast such a partition between kinetic energy (CME), thermal energy (X-ray flare) and energetic particles (SPE). Computing the variation of the magnetic helicity automatically for each AR would give an indication of the onset time of CMEs (NievesChinchilla et al., 2019). We need to better understand all the fundamental solar processes for making progress in deeper understanding the chain of the events.

\subsection{Forecast of ICMEs}

The fast halo CMEs associated with our five X-class flares interact with a previous CME or a streamer that can be identified by multiple shocks and sheaths in each case. All these interactions should lead to geo-effective events (Lugaz et al., 2017). However, in 2002 most of the sources were close to the limb and we measured only the consequences of projection effects. The anomaly of flares occurring mostly at the limb in 2002 leads to the low dip of the geo-effectiveness that year. It is also true for the SPE events which could have been much more energetic and numerous if the flares had occurred from the disk central western part.

The comparison between the existing catalogs revealed that Jian et al. (2006) already identified the five ICMEs in our study. Richardson and Cane (2010) identified the 2 cases \#4 and \#5. Nieves-Chinchilla et al. (2019) identified all the cases except case \#7. In our study, we have identified case \#6 which is not listed in the any other catalogs. Case \#6 has a backside source (E95) and is not associated with an ICME at L1 because of the travel direction of the ejecta. Still the front shock is clearly identified in the interplanetary medium and is associated with a minimum value of the Dst index.

For cases 4 and 5 their two fast halo CMEs (associated with X-flares) coming from the West part of the disk central region are associated with ICMEs at L1 and the geoeffectivity of these events is weak. It demonstrates that forecasting requires either L1 measurements (but with less than 1 hour time delay) or ICME propagation models including magnetic field. 
However, the main unknown is the interplanetary magnetic field configuration during the travel of the ICME and how the ICME shape and direction is altered. We have actually only in situ measurements at L1 given by Wind and ACE spacecraft. Interaction between ICMEs and the heliospheric plasma sheet and current sheet during their propagation can significantly change the ICME/flux rope orientation at different heliocentric distances (Rodriguez et al., 2008; Winslow et al., 2016). Using Messenger, Venus express and ACE spacecraft it has been shown that the solar wind conditions do not recover after the passage of an ICME (Janvier et al., 2019). The knowledge of the interplanetary medium will benefit from the next missions, namely the Parker Solar Probe and Solar Orbiter: we expect to learn more about the shapes of the flux-ropes (magnetic cloud) in the heliosphere, their deformations and erosions with encounters in the solar wind, and their deflections. An armada of small satellites turning around Mercure for example could also provide very suitable in situ measurements. For the forecasts, the numerical simulation models (like ENLIL - Odstrcil and Pizzo (1999)) and EUHFORIA Pomoell and Poedts (2018)) are based on measurements as well and are promising: the simulations compute the interactions involving magnetic reconnection with co-rotating region structures in the solar wind which can alter ICMEs (Winslow et al., 2016). Recent simulations with EUHFORIA can even predict the arrival time of SPEs and ICMEs (Scolini et al., 2018; Wijsen et al., 2019).

\section{Conclusion}

The present paper focus on the $12 \mathrm{X}$-class flares observed in 2002 and observations directly or indirectly related to them (CME, ICME, SPE, indices). With such a small data set, we cannot make proper statistics nor predictions. The novelty of our work is in the study of the details of the Sun-Earth chain of events related to all X-class flares over one year without pre-selection. The main results coming out of this study are that:

- None of the 12 events, even the ones associated with fast halo CMEs from the central disk region, and even with ICMEs and SPE, are geo-effective. It demonstrates that these fast halo CMEs, X-class flares, central disk region, ICMEs, SPEs are not good proxies for geo-effectiveness in 2002. The case studies for the six cases with an associated ICME at L1, coming from different solar longitudes, show for all of them a weak integrated normalized $z$-component of the interplanetary magnetic field, $\mathrm{B}_{z}^{*}$. bf $B_{z}^{*}$ is computed by the integration over time of the IMF $B_{z}$ component, divided by the total interval duration. The weakness of the IMF $\mathrm{B}_{z}^{*}$ explains the very weak geo-effectiveness of these six cases. By the way, as shown Bocchialini et al. (2018), $\mathrm{B}_{z}^{*}$ is more reliable than the commonly used $\mathrm{B}_{z}$ peak value. The peak and sign values are an instant picture of the IMF while $\mathrm{B}_{z}^{*}$ is a more global parameter. A large $\mathrm{B}_{z}^{*}$ implies a long and intense reconnection process at the magnetopause, and increases the probability of having a strong magnetic storm, and a deep Dst decrease.

- For the six events related to an ICME at L1, flare characteristics e.g. longitude and $\min$ (Dst) show a correlation trend. Such a relationship between flares and $\min$ (Dst) would lead to long term prediction (1-3 days). However, due to our small data set, we have to reproduce this study on a larger sample to have a robust conclusion.

- Finally, we offer a complete survey of 12 X-class flares related events in 2002. The only prediction we can make and explain is based on $\mathrm{B}_{z}^{*}$ but L1 observations offer a very short window to raise alerts (less than $1 \mathrm{~h}$ ). The flare duration may give a larger window, but we would need to redo this study on a larger data set to check its robustness.

ANNEX 


\section{A. Halo CME sources in the West side (\#1 and \#10)}

Flare-CME pairs \#1 and \#10 have a source near the limb on the far West side which could explain the weak effect on the Earth environment. Each of them is related to an halo CME followed by ICME and SPE. These are the two most geo-effective events based on Dst and $\mathrm{AE}$ values (Table 1). It is worth noting that it corresponds to the two lowest $\mathrm{B}_{z}^{*}$ values as well. For both events, the weak southward orientation of the IMF is the main explanation, as one can expect $\mathrm{B}_{z}^{*}$ values lower than $-5 \mathrm{nT}$ to observe a stronger Dst depression (Bocchialini et al., 2018). Although flare \#1 has a limb source, the events associated with this flare are the largest ones associated with an X class flare-halo CME in 2002, e.g., the largest CME speed, the largest SPE. However this flare-CME pair \#1 corresponds only to a moderate magnetic storm. For flare-CME pair \#1 the particles are particularly energetic (2520 pfu) in the classification of SPEs. Indices, SW, and IMF observations related to flare/CME \#10 have already been presented (see methodology, section 2.2). The West side was favorable for having a relatively strong acceleration of particles in all the frequency ranges. However the SPE flux for case \#10 is rather low.

\section{B. Halo CME sources in the East side (\#6 and \#7)}

The two flare-CME pairs $(\# 6, \# 7)$ in this group associated with a halo CME have an eastern source. For flare/CME \#6 (X3.3) on July 20, the source was even backside (S13, E95) but the SPE event could be visible two days later on July 21 at 07:00 UT (Figure 9). The SPE event lasts for 30 hours until July 22 12:00 UT. It is consistent with the results in Cane, Reames, and von Rosenvinge (1988) and Reames (1999). This gradual event with long duration from far East can be accounted for by IP shock acceleration (Cane et al., 1988). Podgorny and Podgorny (2018) explained that proton events coming from a source on the East side was due to a transfer across the magnetic field lines with the solar wind. Protons from West side are collisionless particles along the field lines and a delay of several hours is expected. The backside location (behind the eastern limb) of the solar source is also the main explanation for the lack of ICME observations at $\mathrm{L} 1$.

Flare-CME pair \#7 is concomitant to a halo CME: with a very well defined shock front (Figure 10, see the arrows). Figure 11 presents the in-situ measurements at L1 related to flare-CME \#7. Two discontinuities are observed in the magnitude of the IMF (top panel of Figure 11): 24/07 at 07:30 UT and 25/07 at 13:00 UT. Jian et al. (2006) identified an ICME in between the two dashed lines (Figure 11 top panel). We now test the association between the discontinuities and the halo CMEs based on velocity comparisons. As fast ICME shocks are progressively slowing down in the interplanetary medium at least up to $1 \mathrm{AU}$ (Gopalswamy et al., 2000; Grison et al., 2018), we expect $v_{\text {bal }}$ to be lower than the CME velocity $v_{\odot}$ estimated from coronagraph observations and to be larger than the velocity observed at L1 $\left(v_{L 1}\right)$. This is the case for the discontinuity observed the 25 July 13:00 UT (flare-related CME \#6) $\left(v_{L 1}=520 ; v_{b a l}=625 ; v_{\odot}=2285 \mathrm{~km} \cdot \mathrm{s}^{-1}\right)$. The first discontinuity could be connected with the flare-CME \#6. The flare source was indicated as a backside source (S13, E90) but it could be just at the limb. The fast halo CME due to its spherical shape could reach L1 with an unclear ICME signature (indicated by "IP" in Table 1). This velocity could reflect the projected velocity of the expansion of the halo CME or its spherical front (Kwon \& Vourlidas, 2018).

In addition to the $\mathrm{B}_{z}$ IMF component -red curve in the second panel of Figure 11the intensity of southward $\mathrm{B}_{z}(<0)$ is displayed by a color scale in the top panel. Northward intervals are drawn in black and the strongest southward intervals are drawn in blue $\left(\mathrm{B}_{z}<-5 \mathrm{nT}\right.$, see color scale). $\mathrm{B}_{z}$ is the most southward after 26/07 12:00 UT. This can explain why the minimum values of Dst is reached after that time $(27 / 07$ 03:00 UT, cf. blue dot line in the bottom panel) and the weak response of the indices for that event. For flare \#7 no coordinated bump in the SPE records around the time of the X4.8 class on July 23 at 00:42 UT was detected (Figure 9). Either the background was already too 
strong or the flare \#7 was not accompanied by any ejection of protons. Only one - flareCME pair \#6 among the five flare-CME pairs related to SPE events is on the East side of the Sun. The delay between the flares and the particles is long, around 30 hours. This can be explained by the East side location of the source favoring not a good magnetic connection.

C. Halo CME sources near the central meridian (\#4 and \#5)

The two flares \#4, \#5 and their associated halo CMEs have their sources in the same complex active region AR 10030 near the central meridian. The AR is composed of two groups of sunspots (positive leading polarity, negative following polarity for each group) with a strong interaction between them (Figure 2). In fact flare-CME pairs \#4 and \#5 are associated with multi interactive CMEs (Bocchialini et al., 2018). Associated with flare \#4, two CMEs have been identified in the LASCO field of view at 20:30 UT and at 21:30 UT. Their kinetic energy has been estimated to $1.6 \times 10^{32}$ ergs (Table 2). Flare-CME pair \#5 is also associated with two CMEs, a partial CME at 07:31 UT and the halo CME at 08:06 UT.

A convincing ICME signature is observed at L1 for these two events already analyzed by Richardson and Cane (2010) and Bocchialini et al. (2018). The interaction of CMEs leads commonly to high SPE flux (Lugaz et al., 2017). However the SPE flux is low and the value of the $\min$ (Dst) for each event indicates that the geomagnetic storm is also weak. The Dst is equal to $-17 \mathrm{nT}$ and $-36 \mathrm{nT}$ respectively (Table 1 ). Conditions associated with flare-CME pairs \#4 and \#5 (halo CME, fast CME speed, multi CME interaction, ICME at L1, SPE) are usually requested to lead to geo-effective phenomena. We explain the low geo-effectiveness of these events (see AE and Dst columns in Table 1), once again with the weak southward IMF orientation (see $\mathrm{B}_{z}^{*}$ value). Moreover IMF $\mathrm{B}_{z}$ does not display a stable orientation in the ICME sheath following the shock. The particles accelerated (event \#5) between July 19 at 12:00 UT and July 20 at 00 UT are coming from two anti parallel directions which could correspond to the two anchorage feet of the ICME on the Sun. After the 20 July 00 UT, we note only particles at 0 degree at L1 indicating that only a part of the extreme flank of the ICME is crossed. This explains also the long sheath with perturbed $\mathrm{B}_{z}$ (not shown). The delay between the flares and the SPE particles for the two cases (flares \#4, \#5, \#6) are more than 20 hours. For these events the SPEs are suspected to be released in ICME shocks.

\section{Acknowledgments}

B. Schmieder thanks Y.D. Park for his invitation of one month in KASI. We thank the two anonymous referees who help us to focus this study and arrive to a conclusion. The authors thank L. Klein for fruitful discussions on SPEs. This work was supported by the Programme National Soleil-Terre (PNST) of CNRS/INSU co-funded by CNES and CEA. B. Grison acknowledges support of the Czech Science Foundation grant 18-05285S and of the Praemium Academiae Award from the Czech Academy of Sciences. Data analysis was performed with the AMDA science analysis system provided by the Centre de Données de la Physique des Plasmas (CDPP) supported by CNRS, CNES, Observatoire de Paris and Université Paul Sabatier, Toulouse. The data used in this study were obtained from: https://umbra.nascom.nasa.gov/SPE/ for the SPE list (NOAA/Space Weather Prediction Center), http://omniweb.gsfc.nasa.gov/ for the events at L1 (OMNIWeb service), and the CDPP for the events at L1 and the geomagnetic indice http://amda.cdpp.eu (AMDA service). The list of flares and CMEs are available at http://cdaw.gsfc.nasa.gov/CME_list/.

\section{References}

Amari, T., Canou, A., Aly, J.-J., Delyon, F., \& Alauzet, F. (2018, February). Magnetic cage and rope as the key for solar eruptions. Nature, 554, 211-215. doi: 
10.1038/nature24671

Aulanier, G., Démoulin, P., Schrijver, C. J., Janvier, M., Pariat, E., \& Schmieder, B. (2013, January). The standard flare model in three dimensions. II. Upper limit on solar flare energy. Astrophysical Journal, 549, A66. doi: 10.1051/0004$6361 / 201220406$

Aulanier, G., Torok, T., Demoulin, P., \& DeLuca, E. E. (2010, January). Formation of Torus-Unstable Flux Ropes and Electric Currents in Erupting Sigmoids. Astrophysical Journal, 708, 314-333. doi: 10.1088/0004-637X/708/1/314

Bein, B. M., Berkebile-Stoiser, S., Veronig, A. M., Temmer, M., \& Vršnak, B. (2012, August). Impulsive Acceleration of Coronal Mass Ejections. II. Relation to Soft X-Ray Flares and Filament Eruptions. Astrophysical Journal, 755, 44. doi: $10.1088 / 0004-637 \mathrm{X} / 755 / 1 / 44$

Billings, D. E. (1966). A guide to the solar corona.

Bocchialini, K., Grison, B., Menvielle, M., Chambodut, A., Cornilleau-Wehrlin, N., Fontaine, D., ... Zouganelis, I. (2018, May). Statistical Analysis of Solar Events Associated with Storm Sudden Commencements over One Year of Solar Maximum During Cycle 23: Propagation from the Sun to the Earth and Effects. Solar Phys., 293, 75. doi: 10.1007/s11207-018-1278-5

Burlaga, L., Sittler, E., Mariani, F., \& Schwenn, R. (1981, August). Magnetic loop behind an interplanetary shock: Voyager, Helios, and IMP 8 observations. $J$. Geophys. Res., 86(A8), 6673-6684. doi: 10.1029/JA086iA08p06673

Cane, H. V., Reames, D. V., \& von Rosenvinge, T. T. $\quad$ (1988, September). The role of interplanetary shocks in the longitude distribution of solar energetic particles. J. Geophys. Res., 93, 9555-9567. doi: 10.1029/JA093iA09p09555

Cane, H. V., Richardson, I. G., \& von Rosenvinge, T. T. (2010, Aug). A study of solar energetic particle events of 1997-2006: Their composition and associations. Journal of Geophysical Research (Space Physics), 115(A8), A08101. doi: 10.1029/2009JA014848

Carley, E. P., McAteer, R. T. J., \& Gallagher, P. T. (2012, Jun). Coronal Mass Ejection Mass, Energy, and Force Estimates Using STEREO. Astrophysical Journal, 752(1), 36. doi: 10.1088/0004-637X/752/1/36

Chandra, R., Gopalswamy, N., Mäkelä, P., Xie, H., Yashiro, S., Akiyama, S., ... Nitta, N. V. (2013, Dec). Solar energetic particle events during the rise phases of solar cycles 23 and 24. Advances in Space Research, 52(12), 2102-2111. doi: $10.1016 /$ j.asr.2013.09.006

Chiu, M. C., von-Mehlem, U. I., Willey, C. E., Betenbaugh, T. M., Maynard, J. J., Krein, J. A., ... Rodberg, E. H. (1998, July). ACE Spacecraft. Space Science Research, 86, 257-284. doi: 10.1023/A:1005002013459

Cho, K.-S., Marubashi, K., Kim, R.-S., Park, S.-H., Lim, E.-K., Kim, S.-J., ... Lee, J.-O. (2017, April). Impact of the Icme-Earth Geometry on the Strength of the Associated Geomagnetic Storm: The September 2014 and March 2015 Events. Journal of Korean Astronomical Society, 50, 29-39. doi: 10.5303/JKAS.2017.50.2.29

Cid, C., Cremades, H., Aran, A., Mandrini, C., Sanahuja, B., Schmieder, B., ... Zhukov, A. (2012, November). Can a halo CME from the limb be geoeffective? Journal of Geophysical Research (Space Physics), 117(A16), A11102. doi: 10.1029/2012JA017536

Cliver, E. W., Feynman, J., \& Garrett, H. B. (1990, Oct). An estimate of the maximum speed of the solar wind, 1938-1989. Journal of Geophysical Research, 95(A10), 17103-17112. doi: 10.1029/JA095iA10p17103

Cliver, E. W., \& Ling, A. G. (2007, Apr). Electrons and Protons in Solar Energetic Particle Events. Astrophysical Journal, 658(2), 1349-1356. doi: $10.1086 / 511737$

Colaninno, R. C., \& Vourlidas, A. (2009, Jun). First Determination of the True Mass of Coronal Mass Ejections: A Novel Approach to Using the 
Two STEREO Viewpoints. Astrophysical Journal, 698(1), 852-858. doi: 10.1088/0004-637X/698/1/852

Dalmasse, K., Aulanier, G., Démoulin, P., Kliem, B., Török, T., \& Pariat, E. (2015, September). The Origin of Net Electric Currents in Solar Active Regions. Astrophysical Journal, 810, 17. doi: 10.1088/0004-637X/810/1/17

Demoulin, P., Pariat, E., \& Berger, M. A. (2006, January). Basic Properties of Mutual Magnetic Helicity. Solar Phys., 233, 3-27. doi: 10.1007/s11207-006-0010z

Denig, W. F., Wilkinson, D. C., \& Redmon, R. J. $\quad$ (2015, Dec). NOAA Environmental Satellite Measurements of Extreme Space Weather Events. In Agu fall meeting abstracts (Vol. 2015, p. SM32A-01).

Dierckxsens, M., Tziotziou, K., Dalla, S., Patsou, I., Marsh, M. S., Crosby, N. B., ... Tsiropoula, G. (2015, March). Relationship between Solar Energetic Particles and Properties of Flares and CMEs: Statistical Analysis of Solar Cycle 23 Events. Solar Phys., 290, 841-874. doi: 10.1007/s11207-014-0641-4

Echer, E., Gonzalez, W. D., Tsurutani, B. T., \& Gonzalez, A. L. C. $\quad$ (2008, May). Interplanetary conditions causing intense geomagnetic storms (Dst $<=-100$ nT) during solar cycle 23 (1996-2006). J. Geophys. Res.- Space Physics, 113, A05221. doi: 10.1029/2007JA012744

Falconer, D., Barghouty, A. F., Khazanov, I., \& Moore, R. (2011, Apr). A tool for empirical forecasting of major flares, coronal mass ejections, and solar particle events from a proxy of active-region free magnetic energy. Space Weather, 9(4), S04003. doi: 10.1029/2009SW000537

Feng, L., Wiegelmann, T., Su, Y., Inhester, B., Li, Y. P., Sun, X. D., \& Gan, W. Q. (2013, Mar). Magnetic Energy Partition between the Coronal Mass Ejection and Flare from AR 11283. Astrophysical Journal, 765(1), 37. doi: 10.1088/0004-637X/765/1/37

Gonzalez, W. D., Joselyn, J. A., Kamide, Y., Kroehl, H. W., Rostoker, G., Tsurutani, B. T., \& Vasyliunas, V. M. (1994, Apr). What is a geomagnetic storm? J. Geophys. Res., 99(A4), 5771-5792. doi: 10.1029/93JA02867

Gonzalez, W. D., Tsurutani, B. T., McIntosh, P. S., \& Clúa de Gonzalez, A. L. (1996, Jan). Coronal hole-active region-Current sheet (CHARCS) Association with intense interplanetary and geomagnetic activity. Geophysical Research Letters, 23(19), 2577-2580. doi: 10.1029/96GL02393

Gopalswamy, N., Akiyama, S., \& Yashiro, S. (2009, March). Major solar flares without coronal mass ejections. In N. Gopalswamy \& D. F. Webb (Eds.), Universal heliophysical processes (Vol. 257, p. 283-286). doi: 10.1017/S174392130902941X

Gopalswamy, N., Lara, A., Lepping, R. P., Kaiser, M. L., Berdichevsky, D., \& St. Cyr, O. C. (2000, January). Interplanetary acceleration of coronal mass ejections. Geophysical Research Letters, 27, 145-148. doi: 10.1029/1999GL003639

Gopalswamy, N., Yashiro, S., \& Akiyama, S. (2007, Jun). Geoeffectiveness of halo coronal mass ejections. Journal of Geophysical Research (Space Physics), 112(A6), A06112. doi: 10.1029/2006JA012149

Gosling, J. T. (1990). Coronal mass ejections and magnetic flux ropes in interplanetary space. Washington DC American Geophysical Union Geophysical Monograph Series, 58, 343-364. doi: 10.1029/GM058p0343

Grison, B., Soucek, Jan, Krupar, Vratislav, Písa, David, Santolík, Ondrej, Taubenschuss, Ulrich, \& Nemec, Frantisek. (2018). Shock deceleration in interplanetary coronal mass ejections (icmes) beyond mercury's orbit until one au. J. Space Weather Space Clim., 8, A54. Retrieved from https://doi.org/10.1051/swsc/2018043 doi: 10.1051/swsc/2018043

Guennou, C., Pariat, E., Leake, J. E., \& Vilmer, N. (2017, August). Testing predictors of eruptivity using parametric flux emergence simulations. Journal of 
Space Weather and Space Climate, 7(27), A17. doi: 10.1051/swsc/2017015

Hundhausen, A. J. (1997). An introduction. Washington DC American Geophysical Union Geophysical Monograph Series, 99, 1-7. doi: 10.1029/GM099p0001

Ishiguro, N., \& Kusano, K. (2017, July). Double Arc Instability in the Solar Corona. Astrophysical Journal, 843, 101. doi: 10.3847/1538-4357/aa799b

Jang, S., Moon, Y.-J., Kim, R.-S., Kim, S., \& Lee, J.-O. ～(2017, August). Two Distinct Types of CME-flare Relationships Based on SOHO and STEREO Observations. Astrophysical Journal, 845, 169. doi: 10.3847/1538-4357/aa82b4

Janvier, M., Winslow, R. M., Good, S., Bonhomme, E., Démoulin, P., Dasso, S., ... Boakes, P. D. (2019, Feb). Generic Magnetic Field Intensity Profiles of Interplanetary Coronal Mass Ejections at Mercury, Venus, and Earth From Superposed Epoch Analyses. Journal of Geophysical Research (Space Physics), 124(2), 812-836. doi: 10.1029/2018JA025949

Jian, L., Russell, C. T., Luhmann, J. G., \& Skoug, R. M. (2006, December). Properties of Interplanetary Coronal Mass Ejections at One AU During 19952004. Solar Phys., 239, 393-436. doi: 10.1007/s11207-006-0133-2

Kim, R. S., Cho, K. S., Lee, J., Bong, S. C., Joshi, A. D., \& Park, Y. D. $\quad$ (2015, Sep). Characteristics of four SPE groups with different origins and acceleration processes. Journal of Geophysical Research (Space Physics), 120(9), 7083-7093. doi: 10.1002/2015JA021280

Kim, R.-S., Cho, K.-S., Moon, Y.-J., Kim, Y.-H., Yi, Y., Dryer, M., .. Park, Y.-D. (2005, November). Forecast evaluation of the coronal mass ejection (CME) geoeffectiveness using halo CMEs from 1997 to 2003 . Journal of Geophysical Research (Space Physics), 110(A9), A11104. doi: 10.1029/2005JA011218

Kim, R.-S., Park, S.-H., Jang, S., Cho, K.-S., \& Lee, B. S. ～(2017, April). Relation of CME Speed and Magnetic Helicity in CME Source Regions on the Sun during the Early Phase of Solar Cycles 23 and 24. Solar Phys., 292, 66. doi: 10.1007/s11207-017-1079-2

Klein, K.-L., Trottet, G., \& Klassen, A. (2010, May). Energetic Particle Acceleration and Propagation in Strong CME-Less Flares. Solar Phys., 263, 185-208. doi: 10.1007/s11207-010-9540-5

Klein, K.-L., Trottet, G., Samwel, S., \& Malandraki, O. $\quad$ (2011, April). $\quad$ Particle Acceleration and Propagation in Strong Flares without Major Solar Energetic Particle Events. Solar Phys., 269, 309-333. doi: 10.1007/s11207-011-9710-0

Kontogiannis, I., Georgoulis, M. K., Guerra, J. A., Park, S.-H., \& Bloomfield, D. S. (2019, Sep). Which Photospheric Characteristics are Most Relevant to ActiveRegion Coronal Mass Ejections? arXiv e-prints, arXiv:1909.06088.

Kwon, R.-Y., \& Vourlidas, A. (2018, February). The density compression ratio of shock fronts associated with coronal mass ejections. Journal of Space Weather and Space Climate, 8(27), A08. doi: 10.1051/swsc/2017045

Lario, D., \& Simnett, G. M. (2004, Jan). Solar Energetic Particle Variations. In Solar variability and its effects on climate. geophysical monograph 141 (Vol. 141, p. 195). doi: 10.1029/141GM14

Lee, J.-O., Moon, Y.-J., Lee, K.-S., \& Kim, R.-S. ～(2014, June). Dependence of Geomagnetic Storms on Their Associated Halo CME Parameters. Solar Phys., 289, 2233-2245. doi: 10.1007/s11207-013-0466-6

Lepping, R. P., Acũna, M. H., Burlaga, L. F., Farrell, W. M., Slavin, J. A., Schatten, K. H., ... Worley, E. M. (1995, Feb). The Wind Magnetic Field Investigation. Space Science Research, 71(1-4), 207-229. doi: 10.1007/BF00751330

Linan, L., Pariat, É., Moraitis, K., Valori, G., \& Leake, J. (2018, September). Time Variations of the Nonpotential and Volume-threading Magnetic Helicities. Astrophysical Journal, 865, 52. doi: 10.3847/1538-4357/aadae7

Lugaz, N., Temmer, M., Wang, Y., \& Farrugia, C. J. (2017, April). The Interaction of Successive Coronal Mass Ejections: A Review. Solar Phys., 292, 64. doi: 10.1007/s11207-017-1091-6 
Mäkelä, P., Gopalswamy, N., Akiyama, S., Xie, H., \& Yashiro, S. ～(2011, Aug). Energetic storm particle events in coronal mass ejection-driven shocks. Journal of Geophysical Research (Space Physics), 116(A8), A08101. doi: 10.1029/2011JA016683

Manchester, W., Kilpua, E. K. J., Liu, Y. D., Lugaz, N., Riley, P., Török, T., \& Vršnak, B. (2017, Nov). The Physical Processes of CME/ICME Evolution. Space Science Research, 212(3-4), 1159-1219. doi: 10.1007/s11214-017-0394-0

Mays, M. L., Thompson, B. J., Jian, L. K., Colaninno, R. C., Odstrcil, D., Möstl, C., ... Zheng, Y. (2015, Oct). Propagation of the 7 January 2014 CME and Resulting Geomagnetic Non-event. Astrophysical Journal, 812(2), $145 . \quad$ doi: 10.1088/0004-637X/812/2/145

McComas, D. J., Bame, S. J., Barker, P. L., Delapp, D. M., Feldman, W. C., Gosling, J. T., ... Griffee, J. W. (1998, Dec). An unusual coronal mass ejection: First solar wind electron, proton, alpha monitor (SWEPAM) Results from the Advanced Composition Explorer. J. Geophys. Res. Lett., 25(23), 4289-4292. doi: 10.1029/1998GL900174

Menvielle, M., Iyemori, T., Marchaudon, A., \& Nose, M. (2011). Geomagnetic indices. , 183. doi: 10.1007/978-90-481-9858-08

Minnaert, M. (1930, Jan). On the continuous spectrum of the corona and its polarisation. With 3 figures. (Received July 30, 1930). Zeitschrift fr Astrophysik, 1, 209.

Miteva, R., Samwel, S. W., \& Costa-Duarte, M. V. (2018, February). The Wind/EPACT Proton Event Catalog (1996 - 2016). Solar Phys., 293, 27. doi: 10.1007/s11207-018-1241-5

Moon, Y.-J., Choe, G. S., Wang, H., Park, Y. D., Gopalswamy, N., Yang, G., \& Yashiro, S. (2002, December). A Statistical Study of Two Classes of Coronal Mass Ejections. Astrophysical Journal, 581, 694-702. doi: 10.1086/344088

Nieves-Chinchilla, T., Jian, L. K., Balmaceda, L., Vourlidas, A., dos Santos, L. F. G., \& Szabo, A. (2019, Jul). Unraveling the Internal Magnetic Field Structure of the Earth-directed Interplanetary Coronal Mass Ejections During 1995 - 2015. Solar Phys., 294(7), 89. doi: 10.1007/s11207-019-1477-8

Nitta, N. V., Reames, D. V., De Rosa, M. L., Liu, Y., Yashiro, S., \& Gopalswamy, N. (2006, Oct). Solar Sources of Impulsive Solar Energetic Particle Events and Their Magnetic Field Connection to the Earth. Astrophysical Journal, 650(1), 438-450. doi: 10.1086/507442

Odstrcil, D., \& Pizzo, V. J. (1999, Jan). Distortion of the interplanetary magnetic field by three-dimensional propagation of coronal mass ejections in a structured solar wind. J. Geophys. Res., 104(A12), 28225-28240. doi: 10.1029/1999JA900319

Pariat, E., Démoulin, P., \& Berger, M. A. (2005, September). Photospheric flux density of magnetic helicity. Astronomy and Astrophysics, 439, 1191-1203. doi: 10.1051/0004-6361:20052663

Parker, E. N. (1958, Nov). Dynamics of the Interplanetary Gas and Magnetic Fields. Astrophysical Journal, 128, 664. doi: 10.1086/146579

Podgorny, I. M., \& Podgorny, A. I. (2018, November). Proton acceleration in the solar flare. Journal of Atmospheric and Solar-Terrestrial Physics, 180, 9-15. doi: 10.1016/j.jastp.2017.12.010

Pomoell, J., \& Poedts, S. (2018, Jun). EUHFORIA: European heliospheric forecasting information asset. Journal of Space Weather and Space Climate, 8, A35. doi: $10.1051 / \mathrm{swsc} / 2018020$

Reames, D. V. (1999, October). Particle acceleration at the Sun and in the heliosphere. Space Science Research, 90, 413-491. doi: 10.1023/A:1005105831781

Richardson, I. G., \& Cane, H. V. (2010, Jun). Near-Earth Interplanetary Coronal Mass Ejections During Solar Cycle 23 (1996 - 2009): Catalog and Summary of Properties. Solar Phys., 264(1), 189-237. doi: 10.1007/s11207-010-9568-6 
Rodriguez, L., Zhukov, A. N., Dasso, S., Mand rini, C. H., Cremades, H., Cid, C., ... Schmieder, B. (2008, Feb). Magnetic clouds seen at different locations in the heliosphere. Annales Geophysicae, 26 (2), 213-229. doi: 10.5194/angeo-26213-2008

Salas-Matamoros, C., \& Klein, K.-L. （2015, May). On the Statistical Relationship Between CME Speed and Soft X-Ray Flux and Fluence of the Associated Flare. Solar Phys., 290, 1337-1353. doi: 10.1007/s11207-015-0677-0

Sammis, I., Tang, F., \& Zirin, H. (2000, September). The Dependence of Large Flare Occurrence on the Magnetic Structure of Sunspots. Astrophysical Journal, 540, 583-587. doi: 10.1086/309303

Schmieder, B. (2018, November). Extreme solar storms based on solar magnetic field. Journal of Atmospheric and Solar-Terrestrial Physics, 180, 46-51. doi: 10.1016/j.jastp.2017.07.018

Schrijver, C. J. (2007, Feb). A Characteristic Magnetic Field Pattern Associated with All Major Solar Flares and Its Use in Flare Forecasting. The Astrophysical Journal, 655(2), L117-L120. doi: 10.1086/511857

Scolini, C., Messerotti, M., Poedts, S., \& Rodriguez, L. (2018, Feb). Halo Coronal Mass Ejections during Solar Cycle 24: reconstruction of the global scenario and geoeffectiveness. Journal of Space Weather and Space Climate, 8, "A9". doi: $10.1051 / \mathrm{swsc} / 2017046$

Smith, C. W., L'Heureux, J., Ness, N. F., Acuña, M. H., Burlaga, L. F., \& Scheifele, J. (1998, Jul). The ACE Magnetic Fields Experiment. Space Science Research, 86, 613-632. doi: 10.1023/A:1005092216668

Stone, E. C., Frandsen, A. M., Mewaldt, R. A., Christian, E. R., Margolies, D., Ormes, J. F., \& Snow, F. (1998, Jul). The Advanced Composition Explorer. Space Science Research, 86, 1-22. doi: 10.1023/A:1005082526237

Thalmann, J. K., Su, Y., Temmer, M., \& Veronig, A. M. (2015, Mar). The Confined X-class Flares of Solar Active Region 2192. Astrophysical Journal Letters, 801 (2), L23. doi: 10.1088/2041-8205/801/2/L23

Török, T., \& Kliem, B. (2005, September). Confined and Ejective Eruptions of Kink-unstable Flux Ropes. Astrophysical Journal Letters, 630, L97-L100. doi: $10.1086 / 462412$

Tsurutani, B. T., Gonzalez, W., Tang, F., Akasofu, S., \& Smith, E. $\quad$ (1988, Aug). Origin of interplanetary southward magnetic fields responsible for major magnetic storms near solar maximum (1978-1979). J. Geophys. Res., 93(A8), 8519-8531. doi: 10.1029/JA093iA08p08519

Tsurutani, B. T., Gonzalez, W. D., Lakhina, G. S., \& Alex, S. (2003, July). The extreme magnetic storm of 1-2 September 1859. Journal of Geophysical Research (Space Physics), 108, 1268. doi: 10.1029/2002JA009504

Tsurutani, B. T., Gonzalez, W. D., Tang, F., \& Lee, Y. T. (1992, January). Great magnetic storms. J. Geophys. Res. Lett., 19, 73-76. doi: 10.1029/91GL02783

Tsurutani, B. T., Verkhoglyadova, O. P., Mannucci, A. J., Lakhina, G. S., Li, G., \& Zank, G. P. (2009, July). A brief review of solar flare effects on the ionosphere. Radio Science, 44, RS0A17. doi: 10.1029/2008RS004029

Tsurutani, B. T., Wu, S. T., Zhang, T. X., \& Dryer, M. (2003, December). Coronal Mass Ejection (CME)-induced shock formation, propagation and some temporally and spatially developing shock parameters relevant to particle energization. Astrophysical Journal, 412, 293-304. doi: 10.1051/0004-6361:20031413

Vanhamäki, H., Viljanen, A., Pirjola, R., \& Amm, O. (2013, Sep). Deriving the geomagnetically induced electric field at the Earth's surface from the time derivative of the vertical magnetic field. Earth, Planets, and Space, 65(9), 997-1006. doi: 10.5047/eps.2013.03.013

Vasanth, V., Chen, Y., Kong, X. L., \& Wang, B. (2015, June). Investigation of the Geoeffectiveness of CMEs Associated with IP Type II Radio Bursts. Solar Phys., 290, 1815-1826. doi: 10.1007/s11207-015-0713-0 
Vourlidas, A., \& Howard, R. A. (2006, May). The Proper Treatment of Coronal Mass Ejection Brightness: A New Methodology and Implications for Observations. Astrophysical Journal, 642(2), 1216-1221. doi: 10.1086/501122

Vourlidas, A., Patsourakos, S., \& Savani, N. P. (2019, Jul). Predicting the geoeffective properties of coronal mass ejections: current status, open issues and path forward. Philosophical Transactions of the Royal Society of London Series A, 377(2148), 20180096. doi: 10.1098/rsta.2018.0096

Vourlidas, A., Subramanian, P., Dere, K. P., \& Howard, R. A. (2000, May). LargeAngle Spectrometric Coronagraph Measurements of the Energetics of Coronal Mass Ejections. Astrophysical Journal, 534(1), 456-467. doi: 10.1086/308747

Vršnak, B., Sudar, D., \& Ruždjak, D. (2005, June). The CME-flare relationship: Are there really two types of CMEs? Astronomy and Astrophysics, 435, 1149-1157. doi: 10.1051/0004-6361:20042166

Wang, Y., \& Zhang, J. (2007, August). A Comparative Study between Eruptive X-Class Flares Associated with Coronal Mass Ejections and Confined X-Class Flares. Astrophysical Journal, 665, 1428-1438. doi: 10.1086/519765

Wang, Y. M., Ye, P. Z., Wang, S., \& Xue, X. H. (2003, Jul). An interplanetary cause of large geomagnetic storms: Fast forward shock overtaking preceding magnetic cloud. Geophysical Research Letters, 30(13), 1700. doi: 10.1029/2002GL016861

Webb, D. F. (2002, June). CMEs and the solar cycle variation in their geoeffectiveness. In A. Wilson (Ed.), From solar min to max: Half a solar cycle with soho (Vol. 508, p. 409-419).

Wijsen, N., Aran, A., Pomoell, J., \& Poedts, S. (2019, Apr). Interplanetary spread of solar energetic protons near a high-speed solar wind stream. Astronomy and Astrophysics, 624, A47. doi: 10.1051/0004-6361/201935139

Wimmer-Schweingruber, R. F. (2014, January). Interplanetary Disturbances Affecting Space Weather. In B. Schmieder, J.-M. Malherbe, \& S. T. Wu (Eds.), Iau symposium (Vol. 300, p. 297-306). doi: 10.1017/S1743921313011125

Winslow, R. M., Lugaz, N., Schwadron, N. A., Farrugia, C. J., Yu, W., Raines, J. M., .. Zurbuchen, T. H. (2016, Jul). Longitudinal conjunction between MESSENGER and STEREO A: Development of ICME complexity through stream interactions. Journal of Geophysical Research (Space Physics), 121(7), 6092-6106. doi: 10.1002/2015JA022307

Wu, C.-C., \& Lepping, R. P. (2002, Nov). Effect of solar wind velocity on magnetic cloud-associated magnetic storm intensity. Journal of Geophysical Research (Space Physics), 107(A11), 1346. doi: 10.1029/2002JA009396

Xie, H., Gopalswamy, N., Manoharan, P. K., Lara, A., Yashiro, S., \& Lepri, S. (2006, Jan). Long-lived geomagnetic storms and coronal mass ejections. Journal of Geophysical Research (Space Physics), 111(A1), A01103. doi: 10.1029/2005JA011287

Yashiro, S., Akiyama, S., Gopalswamy, N., \& Howard, R. A. (2006, October). Different Power-Law Indices in the Frequency Distributions of Flares with and without Coronal Mass Ejections. Astrophysical Journal Letters, 650, L143L146. doi: $10.1086 / 508876$

Yashiro, S., Gopalswamy, N., Michalek, G., St. Cyr, O. C., Plunkett, S. P., Rich, N. B., \& Howard, R. A. (2004, Jul). A catalog of white light coronal mass ejections observed by the SOHO spacecraft. Journal of Geophysical Research (Space Physics), 109(A7), A07105. doi: 10.1029/2003JA010282

Zuccarello, F. P., Chandra, R., Schmieder, B., Aulanier, G., \& Joshi, R. $\quad$ (2017, May). Transition from eruptive to confined flares in the same active region. Astronomy and Astrophysics, 601, A26. doi: 10.1051/0004-6361/201629836

Zurbuchen, T. H., \& Richardson, I. G. (2006, Mar). In-Situ Solar Wind and Magnetic Field Signatures of Interplanetary Coronal Mass Ejections. Space Science Research, 123(1-3), 31-43. doi: 10.1007/s11214-006-9010-4 

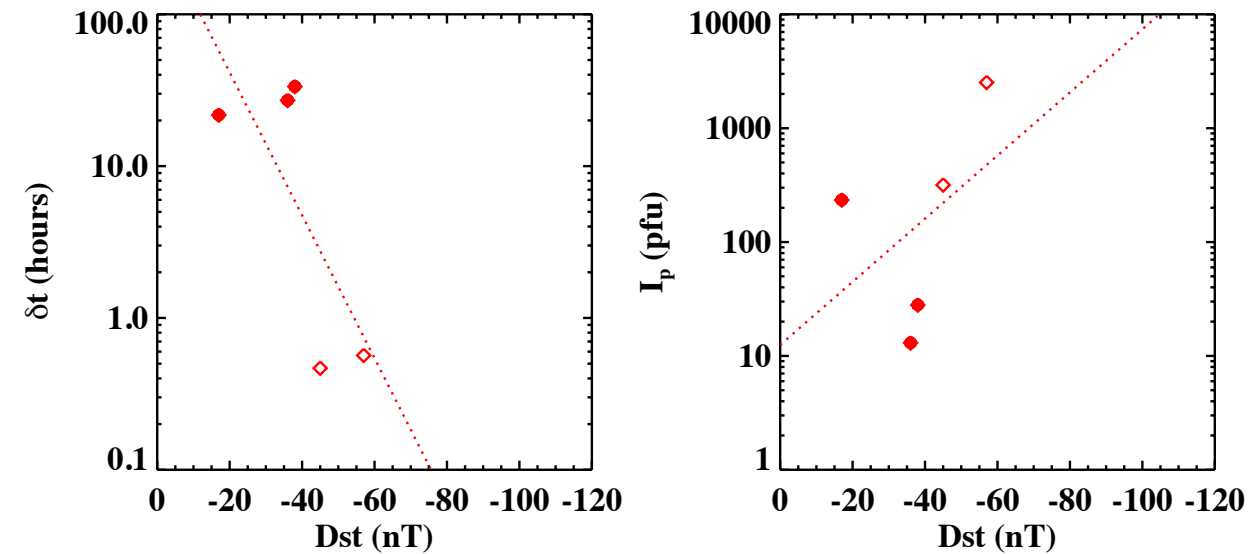

Figure 8. Relationship between SPE characteristics $\left(\delta(\mathrm{t}), \mathrm{I}_{p}\right.$ flux) and Dst. The full/empty red diamonds correspond to long/short SPE-flare delays. 


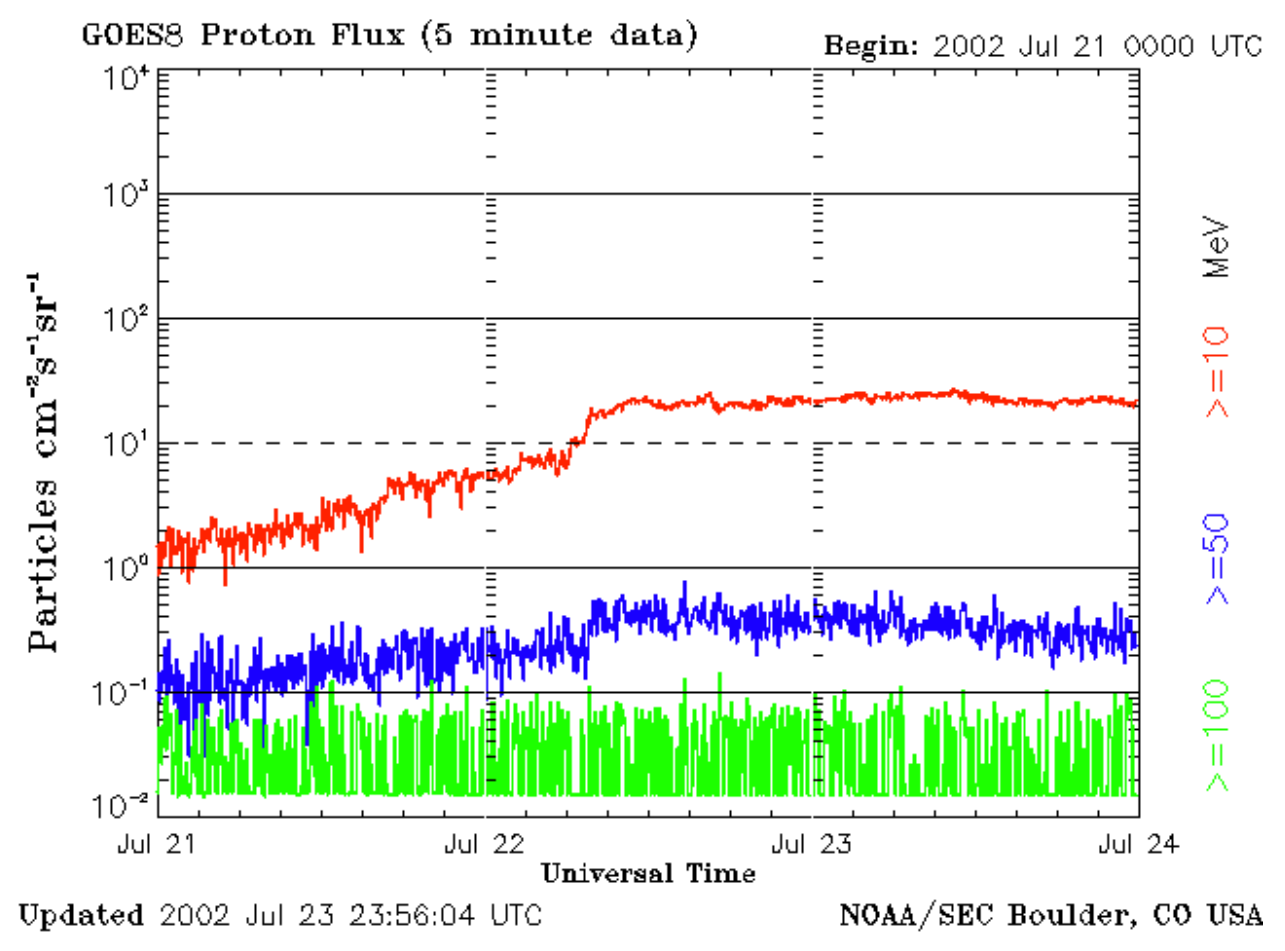

Figure 9. Variation of SPE between July 22 and 27 related to flare \#6. 

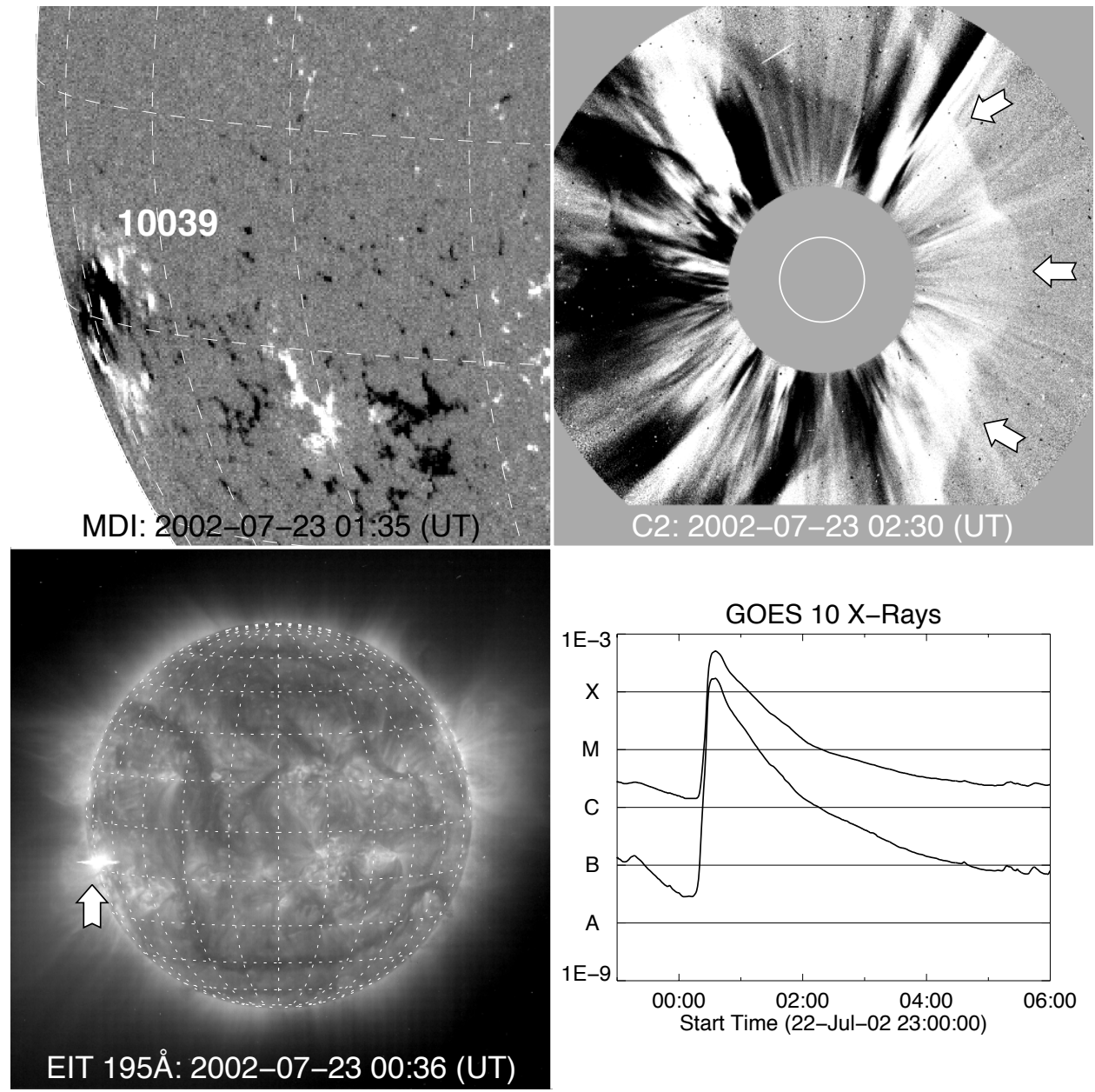

Figure 10. Example of the halo CME source in the East on July 232002 and its solar source (flare \#7). Top panels, left: MDI magnetogram of the flaring active region (AR 10039), right: difference image of the LASCO/C2 coronagraph showing the halo CME and the shock front on the West side, indicated by white arrows. Bottom panels, left: EIT image in $195 \AA$ showing the bright flare (see arrow), right: GOES X-ray record. 


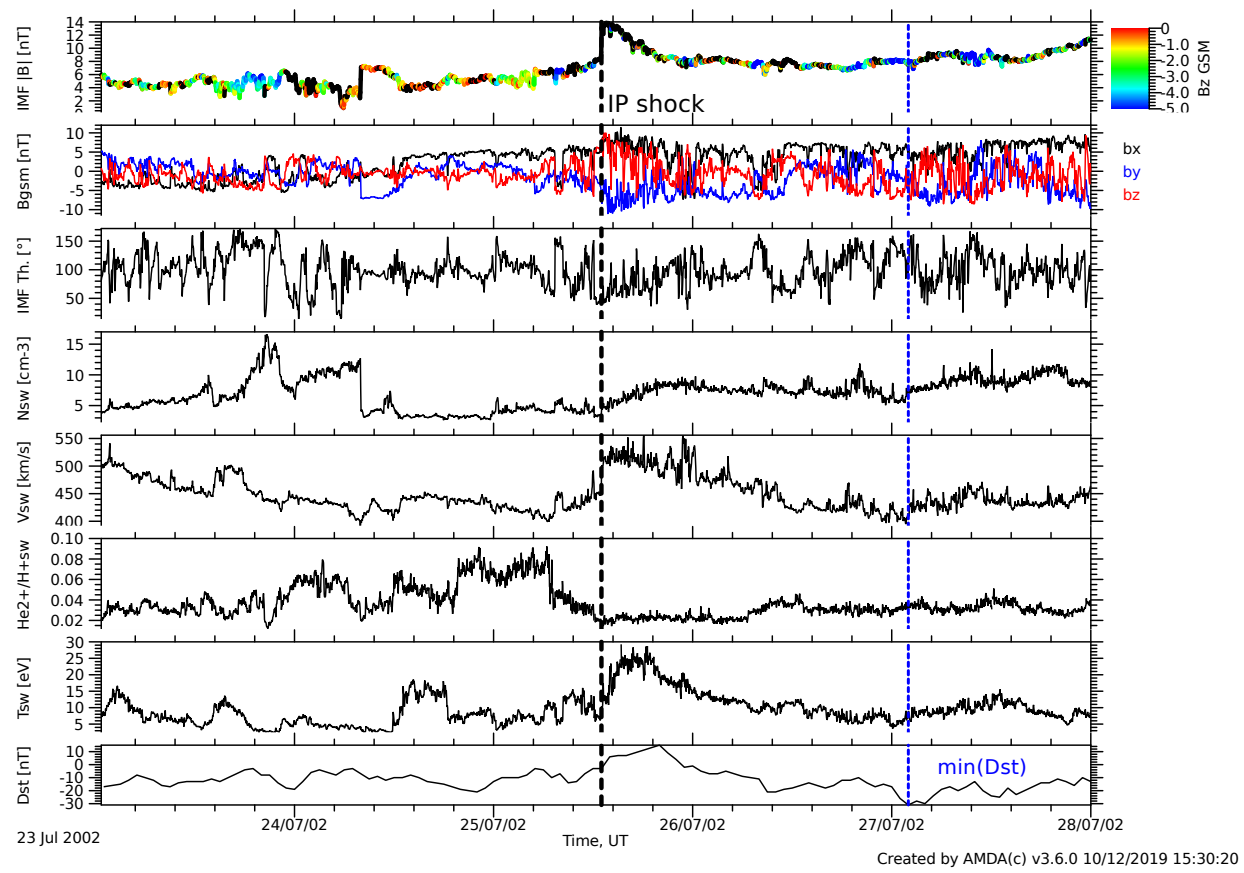

Figure 11. L1 and ground-based observations starting from the 23/07/2002 (time detection of flare \#7) and during 5 days. IMF magnitude, the three IMF components and IMF inclination are plotted in the three top panels (ACE/MAG data). The SW plasma density, flow velocity, radial temperature, and $\alpha$-particle to proton ratio are plotted in the next four panels (ACE/SWEPAM data). In the bottom panel, Dst ground-based index is plotted. the vertical dashed black line indicates the interplanetary shock, the vertical dotted blue line indicates the Dst minimum. 